\title{
Application of Artificial Neural Network in Crop Production: Modeling and Simulation of Plantain Growth Prediction
}

\author{
Dr. Oye N. D. \\ Department of Computer Science \\ Mautech, Yola Nigeria
}

\author{
Adesanya A. A. \\ Department of Computer Science \\ Mautech, Yola Nigeria
}

\begin{abstract}
:
Artificial neural networks (ANN) have become very significant tools in many areas including agricultural system. ANN was used in this research for modeling and simulation of plantain growth prediction. Plantain production is concentrated in the rain-forest belt of West and Central Africa where it constitutes an important staple food of the local population. Using ANN to stimulate the growth of plantain will make plantain farmers to plan their planting. They would already know the outcome of the production and can forecast the financial expenses before and after planting. If they know the height of the plant sucker, (NEL); Leaf width, (LW $(\mathrm{cm}))$; and the Leaf length, (LL(cm)) to predict into future the values of $\mathrm{Ht}, \mathrm{G} 50$, NEL, LW, LL, BW in $\mathrm{kg}$ (Bunch weight), NHB (Number of hands in the bunch) and NFB (Number of fingers in the bunch) of plantain plant based on the planting conditions for dataset used for training in this research. Elman time series neural network, a back-propagation algorithm, with 1 input neuron and 1 output neuron with varying number of neuron in the hidden layer was employed to train the parameters. The network result of Ht, G50, NEL, LW and LL, are used to train the network of BW, NHB, and NFB. Their corresponding coefficient of determination $\left(\mathrm{R}^{2}\right)$ and the Root Mean Squared Error (RMSE) were recorded to determine the acceptance of each network architecture. After all these parameters have been trained, the network predicted into future the value of plantain height, the girth at $50 \mathrm{~cm}$ above the soil level, number of emitted leaves, leaf width, leaf length, bunch weight of plantain, number of hands in the plantain bunch and number of fingers in the plantain bunch given the known value (from the plantain sucker, an experimental value) of Ht, G50, NEL, LW, and LL to the network. It was evidenced that the neural network was able to predict future values of plantain plant and yield at harvest.
\end{abstract}

Key Words: Artificial Neural Network; Modeling; Plantain Growth; Prediction; Simulation.

\section{INTRODUCTION}

\section{Background of the study}

As a productive part of the economy of a country, Agriculture plays a significant role in the national production and Artificial Neural Networks has been used in Agriculture for predicting farm products. Studies have shown that several models and algorithms have been developed to predict yield of agricultural productions and many authors have found linear correlation of yield with soil properties and environmental conditions (Khakural, Robert and Huggins, 1999; Gemtos, Markinos and Nassiou, 2005). However, using nonlinear methods mainly artificial neural networks (ANNs) for yield prediction have become more common (Kominakis,
Abas, Maltaris and Rogdakis, 2002; Kaul, Hill and Walthall, 2005; Sharma, Sharma and Kasana, 2007; Papageorgiou, Markinos and Gemtos, 2011; Papageorgiou, Aggelopoulou, Gemtos and Nanos, 2013). Artificial neural network is a simulation of system of human brain. An interconnected group of artificial neurons that uses mathematical model or computational model for information processing based on the connectionist approach to computation. The interconnection of artificial neurons which may share some properties of biological neural networks, an information processing paradigm that is inspired by biological system and compose of 
interconnection of processing elements (neurons) working together to solve a specific task.

In 1943, a neurophysiologist, Warren McCulloch and a young mathematician, Walter Pitts introduced models of neurological networks, recreated threshold switches based on neurons which showed that even simple types of neural networks could, in principle, calculate any arithmetic or logical function (McCulloch and Pitts, 1943).Hebb (1949) wrote a book entitled "The Organization of Behaviour" which brings the idea that formulated the classical Hebbian rule which represents in its more generalized form the basis of nearly all neural learning procedures. This rule implies that the connection between two neurons is strengthened when both neurons are active at the same time. This change in strength is proportional to the product of the two activities.

Many researchers (Rosenblatt, 1961; Minsky and Papert, 1969) worked on perceptron. The neural networks can be proved to adjust to the correct weight that will solve the task. Minsky and Papert showed that perceptron could not learn those functions which are not linearly separable and opined that neural network research should be discredit and divert neural network research funding to the field of "Artificial Intelligence" and so put an end to overestimation, popularity and research funds. After Minsky and Papert's demonstration of the limitations of perceptrons, research on neural network still continued but there were neither conferences nor other events and therefore only few publications. Among those that published in the 1970s include Malsburg (1973), Werbos (1974), Grossberg (1976), Hopfield (1982) and Fukushima, Miyake, and Ito (1983). These people among other researchers were the people who put the field of neural network on a strong expectations and prepared the lead for the renaissance of the field.
Through the work of John Hopfield, with his lectures, encouraged hundreds of highly qualified scientists, mathematicians, and technologists to join the emerging field of neural networks and the wide publication of backpropagation by Rumelhart, Hinton and Williams (1986). The field exploded and nonlinearly separable problems could be solved by multilayer perceptrons, and this disproved Marvin Minsky's negative evaluations. After this time, the development of neural network has almost been explosive. The economic significance of plantain cannot be over emphasized, its health uses, and it forms significant part of the diets of people living in the rain-forest belt. In this research, Artificial Neural Network was used for modeling and simulation of plantain growth development, the morphology growth of plantain and yield with some certain criteria as identified parameters.

Plantain production is concentrated in the rain-forest belt of west and central Africa where it constitutes an important staple food of the local population. Plantains (Musa paradisiaca) are potent sources of micronutrients especially vitamins $\mathrm{A}$ and $\mathrm{C}$, potassium and fibre. A firm ripe plantain have high carbohydrate content, a good sources of vitamins and minerals in addition to being low in fat (Yusufu, Mosiko and Ojuko, 2014). Musa species are useful as food to be consumed by human either as flour to be used in confectionaries or as jams and jellies; in chips etc. Its peel can be used as animal feed. All parts of the plantain plant have medicinal applications: the flower in bronchitis and dysentery and on ulcers, cooked flowers are given to diabetics etc. Its leaves are also useful for lining cooking pots and for wrapping. Improved processes have also made it possible to utilize banana fibre for ropes, table mats and handbag (Chandler, 1995).

Ngo-Samnick, E.L. (2011) discussed the agronomic requirements for planting a plantain as:

Temperature: The optimal temperature for growing plantain is $28^{\circ} \mathrm{C}$. From $28^{\circ} \mathrm{C}$ to $20^{\circ} \mathrm{C}$, growth will 
gradually slow down, and will become negligible around $16^{\circ} \mathrm{C}-18^{\circ} \mathrm{C}$.

Light: Shade accelerates height growth, and it is advisable to determine the density depending on the cultivar selected, in order to provide the best light conditions for the plantation.

Water: Plantain needs a lot of water. It should get around $200 \mathrm{~mm}$ per month throughout its life cycle. Wind: Plantain is very sensitive to strong wind, which can cause physical damage to the plant (torn leaves, toppling). Soil: Plantain grows best in deep soil that is well drained and rich in organic matter. Fallow land can also be used, but the yield will depend on what was previously grown and the duration of the fallow.

\section{Statement of the Problem}

Plantain farmers experience low production due to lack of proper planning, sometimes fail to put good measure in place that will increase their farm productivities. Poor preparation before the start of production will bring low yield of crop which also will lead to waste of resources in material and man effort. Using Artificial neural network to stimulate the growth of plantain development will make plantain farmers to plan their planting, they would already know the outcome of the production and can forecast the financial expenses before and after planting which will reduce the waste of resources and increase the agricultural economy of the country. With this scientific approach, the plantain farmers can predict the growth and yield of the plant if they know the Pseudostem height of the plant sucker, Ht $(\mathrm{cm})$; Pseudostem girth at $50 \mathrm{~cm}$ above soil level, G50 (cm); Number of emitted leaves on the sucker, NEL; Leaf width, LW (cm); and the Leaf length, $\mathrm{LL}(\mathrm{cm})$ to predict into future the values of Pseudostem height, $\mathrm{Ht}(\mathrm{cm})$; Pseudostem girth at $50 \mathrm{~cm}$ above soil level, G50 (cm); Number of emitted leaves, NEL; Leaf width, LW (cm); Leaf length, LL (cm); Bunch weight, BW (Kg); Number of hands in the bunch, NHB and Number of fingers in the bunch, NFB of plantain plant based on the planting conditions for dataset used for training for this research

\section{Aim and Objectives of the Study}

This research modeled and simulated the growth of plantain development using artificial neural network and the identified objectives are:

i. Developed a three-layer network Architecture using Error correction learning algorithm.

ii. developed a time series Elman neural network code and simulate the sample data collected for the model using MATLAB software.

iii. calculated the output of the network and compare to the corresponding target vector.

iv. measured at every stage of iteration, the difference between the target value and the experimental value was maintained in other to keep control until target value was reached.

v. the weights of the neurons are adjusted according to the algorithm, backpropagation which tends to minimize the error, to produce the desired output.

vi. Calculated RMSE to verify how concentrated the data around the line of best fit.

\section{Justification and Significance of the Study}

This study is significant in that it:

1. Allowed the farmers to have better conditions for planning, maximizing the efficiency of the business process without loses because it will provide reliable estimates for the optimal time of a harvest that will lead to increased shelf life. 
2. Plan financing aspects of production before and after planting and this encourage the continuation of studies to verify which variables most influence on the final production. This will improve the agricultural economy of Nigeria if practice and however also reduce waste of resources available.

\section{LITERATURE REVIEW}

\subsection{Application Areas of Artificial Neural Networks in Agriculture}

Artificial neural networks have become very significant tools in many areas including agricultural system. Agricultural system is a complex system since it deals with a number of factors with a large data situation. Different techniques and approaches have been adopted to show any interactions between factors that is affecting yields with plant growth. Many researchers have shown the application of artificial neural networks (ANNs) in agriculture (Diamantopoulou, 2005; Movagharnejad and Nikzad, 2007; Zhang, Bai and Liu, 2007). Most of these studies were dedicated to the predictions of yield. (Jiang, Jiang, Yang, Clinton and Wang, 2004) showed in their work using ANNs model with the back-propagation training algorithm to forecast the yield in wheat winter crops by using remote sensing information. (Uno, Prasher, Lacroix, Goel, Karimi, Viau and Patel, 2005) developed models for the prediction of yield in maize, by using statistical methods and ANNs with various indexes of vegetation; the greater accuracy in the prediction was obtained with the ANNs model, which was superior to any of the three conventional empirical models.

\subsection{ANN for Modeling in Agriculture}

Zee and Bubenheim (1997) developed a plant growth (physiology) model using artificial neural networks. In their approach, they identified four crop processes to crop-base life support systems operation which were identified as Assimilation, Allocation, Nutrient Uptake and Transpiration. Taken together these four processes result in a complete life support system package, can be highly reliable and controllable. They presented the modeling of a single plant process of transpiration (water production) with varying inputs of air temperature, canopy temperature, relative humidity, and plant type. In their report, they discussed four different mathematical models for transpiration during crop growth which are: flow model using Ohm's law, transport flow model, radiation balance, and mass flux. In their conclusion, they identified the major challenge for implementing artificial neural network model as large amounts of training data required over a range of environmental conditions and plant growth experiments usually require several weeks to several months, and usually very few data are recorded.

Bala, Ashraf, Uddin and Janjai, (2005) presented experimental performance and artificial neural network modeling of drying of litchi flesh in a parabolic greenhouse solar dryer. The dryer consists of a parabolic roof structure covered with polycarbonate sheets on a concrete floor. An artificial neural network (ANN) approach was used to model the performance of the dryer for the drying of litchi flesh. Using solar drying data of litchi flesh, the ANN model was trained using the backpropagation algorithm with seven sets of data used for training and three sets were used for testing the ANN model. Ten tests of the dryer for the drying of litchi flesh were performed during three harvest seasons of litchi, namely 2008, 2009 and 2015. They showed the comparison of air temperatures at three different locations inside the dryer, namely front, middle and back, for a typical experiment of solar drying litchi flesh. Temperatures at different 
positions in these three locations varied within a narrow band. The airflow rate increases sharply in the early part of the day, then becomes fairly constant and then drops sharply in the afternoon. They concluded that solar radiation followed similar patterns for all days during drying. Moisture content of the litchi flesh was reduced from an initial value of $84 \%(\mathrm{wb})$ to the final value of $13 \%$ (wb) within 3 days. The dryer can be used to dry up to $100 \mathrm{~kg}$ of litchi flesh.

Liu, He, Song, and Yang (2010) developed a prototype system to verify the feasibility and effectiveness of structure and function of plant growth model. For different characteristics of plant growth, and supply convenience for the appearance of the whole plant such as texture, light and color and so on, changing properties of relevant organs and physiological function parameters, the process of simulated results can be obtained. The unilateral light of long-term effects is caused by trendreaction, resulting in plant morphology of bending to the bright side, reflected in the uniform of light intensity to the role of the top growth characteristics. The plant shows malformation growth under strong winds, leading to change of plant structure. The simulation of plant growth process shows that the physiological characteristics of plants will respond accordingly and lead to the change of plant morphology and topology according to the rules when light, temperature, moisture and nutrients that affect plant growth and other conditions change during the process between the beginning and maturation.

\subsection{ANN for Prediction in Agriculture}

Qiao, Shi, Pang, Qi, and Plauborg (2010) estimated the water uptake by plant roots with artificial neural network. Seven factors were used as input parameters which were soil moisture, potential evapotranspiration, atmospheric humidity, electrical conductivity of soil solution, air temperature, plant shoot height and diameter while root water uptake rates at different depth in the soil profiles were taken as the outputs. Root capability to extract water from soil depends on both soil and plant properties. Determination of volume, conformation and distribution of the roots in soil poses a lot of difficulties to scientists, since non-invasive methods for explicit description of the whole plant root system have not yet been elaborated. They used a feed-forward three layers neural network model and this model effectively came out with a small relative error, which was less than $17 \%$.

Soares, Pasqual, Lacerda, Silva, and Donato (2013) proposed a methodology for predicting the bunch's weight in banana plants. The implemented ANN produced an efficient prediction of the production. The networks were trained with experimental data representing the characteristics of the growth and development of the plant in order to obtain the production, which can achieve greater ability to generalize since more data is supplied to the network for training. The data from the first cycle were used to build the prediction model and data from the second cycle were reserved to test the predictive capability of the model. Safa, Samarasinghe, and Nejat (2015) showed in their study the first time an ANN model was designed to predict agricultural production using direct factors as well as indirect factors, and it showed the potential of using indirect factors to predict agricultural production (wheat production of farm inputs using indirect parameters). The final neural network model, using a carefully selected set of six inputs from more than 140 different factors, can predict wheat production based on wheat area, irrigation frequency, machinery condition (tractor hp ha- and number of passes of sprayer), and farm inputs $(\mathrm{N}$ 
and fungicides consumption) in Canterbury arable farms with an error margin of $\pm 9 \%\left( \pm 0.89 \mathrm{t} \mathrm{ha}^{-1}\right)$. The results of their study showed the ability of ANN models to predict wheat production using heterogeneous data better than using a multiple regression model (as a common model used in agricultural studies).

\section{METHODOLOGY}

\subsection{Neural Networks Overview and Heuristics}

Basic Concepts of Neural Networks

The "Neural Network" as employed in this research is obviously not a biological concept, it is artificial. In the field of neural networks, "Neural Networks" and "Artificial Neural Networks" are interchangeable. Neural networks are inspired by the functioning of the biological network of neurons in human brain. A human brain contains approximately $10^{11}$ computing elements called neurons. Those neurons are fully connected and communicate with each other. The inputs are received by the sensory receptors and sent to the neurons in the network. The neurons process the inputs and send information to the next neurons (Zurada, 1992).

A neuron is nothing more than a switch with information input and output. The switch will be activated if there are enough stimuli of other neurons hitting the information input. Then, at the information output, a pulse is sent to other neurons. Incoming signals from other neurons or cells are transferred to a neuron by special connections, the synapses. Such connections can usually be found at the dendrites of a neuron, sometimes also directly at the soma (cell body).

\subsection{Computational Model of Human Neuron}

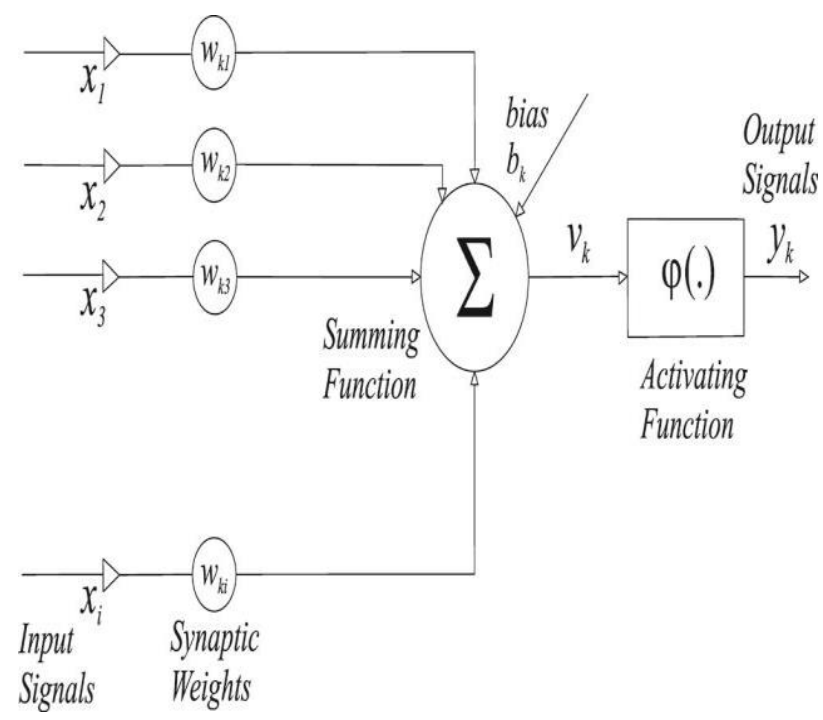

Figure 3.1: Design of a simple Artificial Neural

Network with $\mathrm{i}$ input variables and $\mathrm{k}$ neurons in its output layer (modified after Aji et al. 2013)

Figure 3.1 shows the artificial neuron model. The sets $x_{1}, x_{2}, \ldots x_{i}$ represent input signals (for example in agriculture; leaf water content, nutrients, soil quality and other agricultural factors), the $w_{k i}$ are synaptic weights (The strength of the neuron) which indicate the connection weight from neuron $\mathrm{j}$ to neuron $\mathrm{k}, b_{k}$ is a bias (which can increase the adaptability of neurons and neural networks.) from neuron $\mathrm{k}, v_{k}$ is an activation potential of the neuron $\mathrm{k}, \varphi($.$) is an activation function, y_{k}$ is the output signal of the neuron $\mathrm{k}$ and $u_{k}$ is the net input, which is the sum of all inputs multiplied by all synaptic. The output is of the form $u_{k}=\sum_{j=1}^{i} w_{k j} x_{j}$ 1 and the combined output, $v_{k}=u_{k}+b_{k}$ where $y_{k}=\varphi\left(u_{k}+b_{k}\right)$ 
The Threshold Function

If $v_{k} \geq 0, y_{k}=1 \quad$ otherwise, $y_{k}=0$

The response graph is:

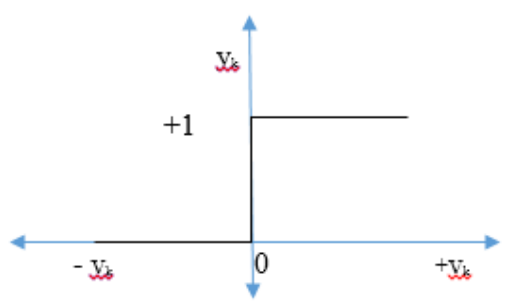

Applying the activation function $\varphi($.$) So that$ equation turns to non-leaner function, sigmoid function is applied.

$\varphi\left(v_{k}\right)=\frac{1}{1+\ell^{v_{k}}}$

The response graph is

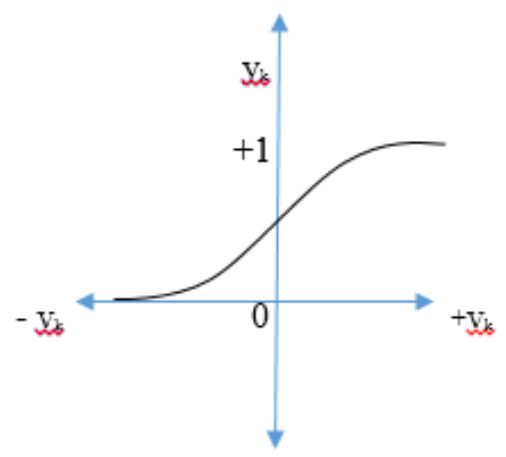

\subsection{Architecture of the Neural Network}

The architectural structure is a three-layered Elman time series neural network as depicted in the figure 3.2. The input layer of Neurons are the inputs to the network. Elman network is a feedback neural network that has the weights of the first layer coming from the input and each subsequent layer has a weight coming from the previous layer. All layers except the last one have a recursive weight and the last layer is the network output.

Elman network use a memory called content unit (q) to store the values of the hidden layer outputs and feedback these values to the network for retraining.

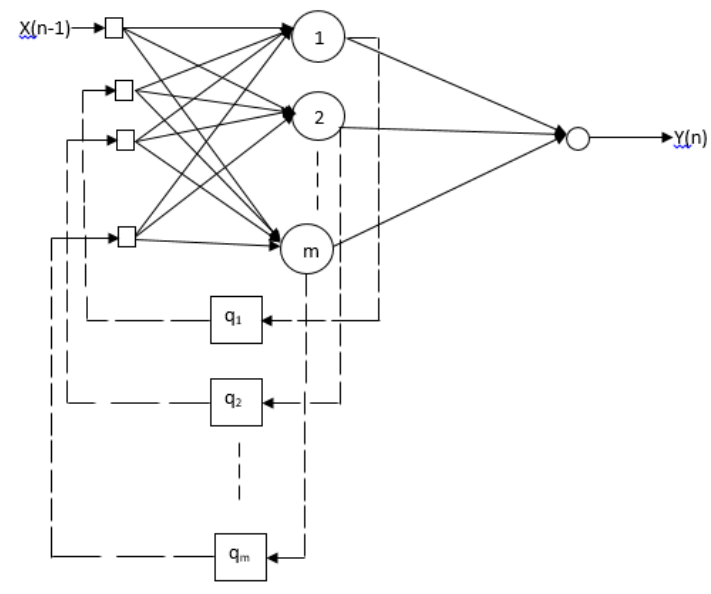

Figure 3.2: A Time series Elman neural network with one input, 1 hidden layer of $m$ neurons and one output.

\subsection{Data Collection and Analysis}

The dataset used for this work was the 'PlantainOptim' dataset, from the 'Plantain-Optim' experiment conducted from 2009 to 2011 at CARBAP experimental station in Cameroom (Sylvain, Frédéric, Médard, Désirée, David, JeanPierre, Bernard and Thierry, 2018). The column of data considered from this dataset are Days after planting, DAP; Pseudostem height, Ht (cm); Pseudostem girth at $50 \mathrm{~cm}$ above soil level, G50 (cm); Number of emitted leaves, NEL; Leaf width, LW (cm); Leaf length, LL (cm); Bunch weight, BW $(\mathrm{Kg})$; Number of hands in the bunch, NHB and Number of fingers in the bunch, NFB which are the identified parameters considered for the neural network used for this work.

From the 'Plantain-Optim' experiment, each leaves emitted on individual plant were numbered and measured. The leaf width and leaf length were taken into account for each emitted leaf. However for this work, the leaf width and leaf length assumes the value of the emitted number of leaves at every instance of time interval of 15-day accuracy of 
record for the 'Plantain-Optim' dataset. For instance, if the emitted number of leave is 7 at a particular time in days, the value of leave width and leave length was the leave width and leave length of the 7 th leave of the plant. The dataset were recorded every 15 days interval and the first observation of data recorded was at 35 days from the first day of planting.

From the 'Plantain-Optim' dataset, it was observed that some plants do not have values at the harvest period which make those plants not usable or considered to be used for the neural network prediction. It was also noticed that there are off set in harvest period. Some plants matured in 10 months, some are in 11 months, 12 months, 13 months and 14 months. The dataset are categorised based on this harvest periods and 11 months plants were selected to limit the error variation of values predicted by the neural network result because plants that fall in this category are much and the more the dataset, the better the neural network's result. However, due to time factor to format the dataset in the specific arrangement that the network can access it properly, only 51 dataset are used, containing of 5 varieties of plantain plant data.

The dataset was prepared for a time-series multi-step prediction network. The number of days of sampling of the data was interpolated to decrease sampling period from 15 days to 1 day as opposed every 15 days in the 'Plantain-Optim' dataset. The MATLAB code is given below:

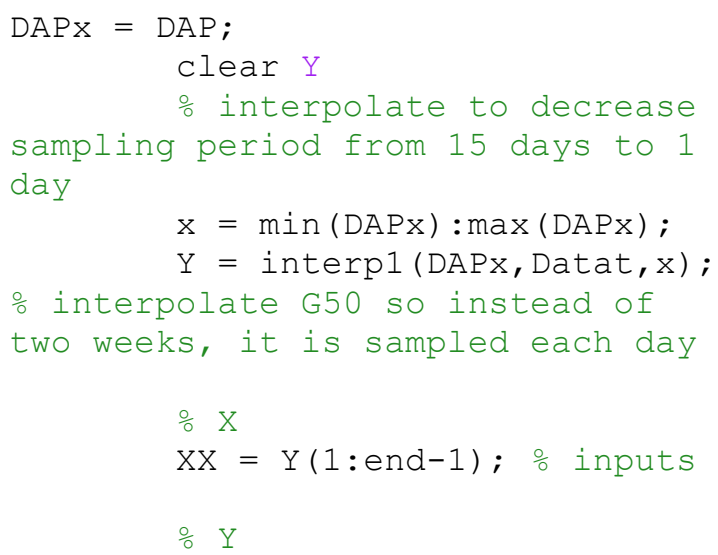

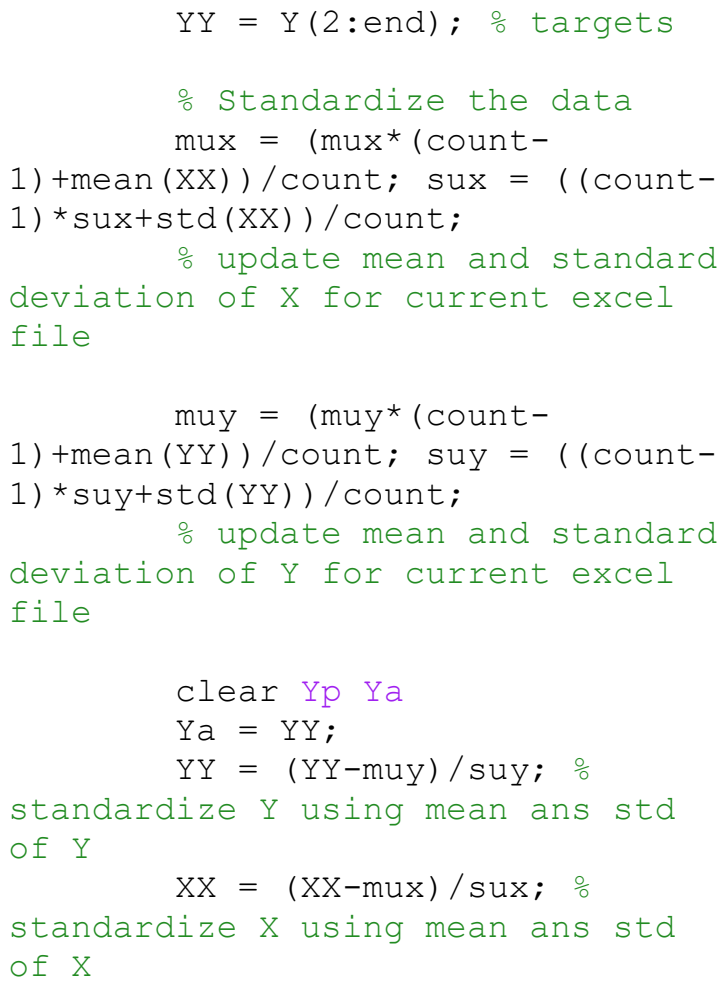

\subsection{Plantain Variety}

There are many varieties of plantain in existence by nature, nine varieties were experimented at CARBAP experimental station in Cameroom, which are Batard, Big Ebanga, CRBP39ª (Hybrid plant), D248 (Hybrid plant), D535 (Hybrid plant), Essong, French Clair, FHIA21 ${ }^{\mathrm{b}}$ (Hybrid plant) and Mbouroukou $n^{0} 3$. Forty-five numbered elementary experimental plots were used and each experimental plot included nine plants of one variety. Each variety was represented by 45 plants.

\subsection{Planting Conditions}

This work adopted all the planting conditions that were put in place (including climate conditions, water percentage and soil texture) for the 'PlantainOptim' dataset for the result of the neural network to be accepted.

\subsection{Input and Output}

In this case, a time series neural network with an input layer, a hidden layer of $\mathrm{m}$ neurons (this is different for each network parameter) and an output layer is used for Pseudostem height, $\mathrm{Ht}(\mathrm{cm})$; 
Pseudostem girth at $50 \mathrm{~cm}$ above soil level, G50 (cm); Number of emitted leaves, NEL; Leaf width, LW (cm) and Leaf length, LL (cm).

For number of neurons in hidden layers, there is no hard and fast rule to compute that for optimal architecture of the network (Khazaei, Tavakoli, Ghassemian, Khoshtaghazaa, Banakar, 2013), either most of the time, one just go with what works best to have a good result from the network prediction by cross validation. The number of neurons in input layer always equals the number of features considered. One (1) input neuron in the case for $\mathrm{Ht}$, G50, NEL, LW and LL since we are treating each parameter as time series, so the current value is predicted by past values while the neurons in the hidden layer differ for each identified parameter and the neuron in the output layer is 1 but for Bunch weight, BW $(\mathrm{Kg})$; Number of hands in the bunch, NHB and Number of fingers in the bunch, NFB their number of inputs are 5 (based on the output generated for the values from Ht, G50, NEL, LW and LL), a hidden layer with $\mathrm{m}$ neurons and 1 output neuron in the output layer since we only need one output.

BW, NHB and NFB has no much dataset for training neural network as lots of dataset is required by artificial neural network to predict very accurately, for that reason, the networks were trained over and again for 30 times to have better result and their hidden neurons were changed (see Table 4.7, Table 4.8 and Table 4.9) to verify the best architecture for each network. Table 4.7 shows the architectures and statistical parameters for artificial neural networks tested for bunch weight, Table 4.8 shows the architectures and statistical parameters for artificial neural networks tested for number of hand in the bunch and Table 4.9 shows the architectures and statistical parameters for artificial neural networks tested for number of fingers in the bunch.

\subsection{Training Algorithm}

For the Ht, G50, NEL, LW and LL, the networks were trained with Gradient decent with momentum and adaptive LR (trangdx) training algorithm with data division as random (dividerand), using the default ratio, $70 \%$ of dataset for training, $15 \%$ of dataset for validation and $15 \%$ of dataset for test. Mean square error (MSE) was used as performance measure.

For the BW, NHB and NFB, the artificial neural networks use an Input-Output Fitting Neural Network and the networks were trained with trainlm algorithm. Trainlm is a network training function that updates weight and bias values according to Levenberg-Marquardt optimization. It is often the fastest backpropagation algorithm in the Matlab toolbox, and is highly recommended as a firstchoice supervised algorithm, although it does require more memory than other algorithms.

RMSE (Root Mean Squared error) was used to verify how concentrated the data is around the line of best fit. It shows how much the predicted values deviate from the experimental values in the data set on average. The metric unit is the same unit as its variable.

The network was trained on normalized values, so once the predictions is done, the output is also normalized but the actual values (the experimental values) which are subtracted from the predicted values to get RMSE are not normalized, therefore, the output was de-normalize as well.

So in this case: $\mathrm{Yp}=\operatorname{cell} 2 \mathrm{mat}(\operatorname{net}(\mathrm{X}, \mathrm{Xi}, \mathrm{Ai}))$; and $\mathrm{Yp}$ $=$ Yp*suy +muy;

$\mathrm{Yp}=\operatorname{cell} 2 \mathrm{mat}(\operatorname{net}(\mathrm{X}, \mathrm{Xi}, \mathrm{Ai}))$; gives the normalized (or standardized) output

$\mathrm{Yp}=\mathrm{Yp}$ * $^{*}$ suy +muy; scales the output to the original values where suy is the standard deviation of output data used for standardization of actual (experimental) output and muy is the mean of output data. 
After the first training was done, the network was trained further for nine times to reduce the amount of error generated by employing Monte Carlo simulation to repeat the same experiment again and again and then taking average.

\subsection{Algorithm for Training Network}

The basic algorithm loop structure of Backpropagation algorithm

Initialize the weights

Repeat

\section{For each training pattern}

"Train on that pattern"

End

Until the error is acceptably low.

The learning method is a supervised learning, there is a set of training samples and neural network adjusts its connection weights according to the difference between predicted outputs and experimental outputs.

The learning algorithm: Error correction learning law

$$
e_{i}(t)=Y p_{i}(t)-Y a_{i}(t)
$$

Where $Y p_{i}(t)$ is the predicted output of ith neuron at time $\mathrm{t} ; \mathrm{Ya}(t)$ is the actual output of ith neuron at time t and $e_{i}(t)$ is the output error of ith neuron at time t. The goal is to minimize some function of $e_{i}(t)$.

\subsection{System Requirement}

The MATLAB software version used was MATLAB R2015a and the system configuration used to carry out this is HP 250 with Windows 8.1 running on it as operating system.

System processor: Intel(R) Pentium(R) CPU B960 (a) $2.20 \mathrm{GHz}$

System installed memory (RAM): 4.00GB 3.89GB (usable)
System type: 64-bit Operating system, X64-based processor

Hard drive: 500GB

\subsection{Time delay Neural Network, an option to} Elman Neural network

Time delay networks are similar to feedforward networks, except that the input weight has a tap delay line associated with it. This allows the network to have a finite dynamic response to time series input data with the availability of full dynamic derivative calculations (fpderiv - Forward propagation derivative function and bttderiv - Backpropagation through time derivative function). Elman networks are feedforward networks (feedforwardnet) with the addition of layer recurrent connections with tap delays. Elman networks with one or more hidden layers can learn any dynamic input-output relationship arbitrarily well, given enough neurons in the hidden layers. However, Elman networks use simplified derivative calculations (using staticderiv - Static derivative function, which ignores delayed connections) at the expense of less reliable learning (Mathworks, 2018).

\section{RESULTS PRESENTATION AND}

\section{ANALYSIS}

\subsection{Training Analysis}

Fig. 3.2 shows the architecture of the neural networks developed by using the 'backpropagation' algorithm for the prediction of the plantain plant growth. The networks were trained with experimental data obtained from the 'PlantainOptim' dataset, from the 'Plantain-Optim' experiment conducted from 2009 to 2011 at CARBAP experimental station in Cameroom, representing the characteristics of the growth and development of plantain plant. The dataset used are 11 months harvest period of different varieties of 
plants as discussed in the methodology. The results of this process are described below. Since this is a time series neural network, each parameters identified as plant growth parameters were trained separately. Each of the training sections was carried out with different initial weights and hidden layer neuron from 1 to 10 to know the best architecture for each network, each network were trained over and again for 10 times and put under observation so that the data will not over fit until the lowest average error was obtained. The network with the lowest RMSE (root mean squared error) was selected.

Table 4.1, explains the architectures and statistical parameters for ANNs tested for $\mathrm{Ht}(\mathrm{cm})$ and the best neural network architecture for $\mathrm{Ht}(\mathrm{cm})$ prediction model was made up of a network with architecture of $1: 3: 1$. Table 4.2 explains the architectures and statistical parameters for ANNs tested G50 (cm) and the best neural network architecture for G50 (cm) prediction model is $1: 2: 1$. Table 4.3 explains the architectures and statistical parameters for ANNs tested for NEL and the best neural network architecture for NEL prediction model is 1:3:1. Table 4.4 explains the architectures and statistical parameters for ANNs tested for LW (cm) and the best neural network architecture for LW (cm) prediction model is 1:2:1 and while Table 4.5 explains the architectures and statistical parameters for ANNs tested for LL $(\mathrm{cm})$, and the best neural network architecture for NEL prediction model is $1: 9: 1$.

Table 4.1, Table 4.2, Table 4.3, Table 4.4 and Table 4.5 summaries the characteristics and the parameters of the tested artificial neural networks for each variable parameter, which are: Network Architecture, Min. Performance gradient, Max. Number of epoch trained, Learning rate ( $\eta$, Performance goal, $\mathrm{R}^{2}$ and RMSE. The predicted values by the ANNs against the experimental data were used to calculate the coefficients of determination $\left(\mathrm{R}^{2}\right)$ and the Root Mean Squared Error (RMSE). It was observed from all the architectures and statistical parameters for ANNs tested tables that when the RMSE reduces, the $\mathrm{R}^{2}$ will increase and where the RMSE increases, the values of the $\mathrm{R}^{2}$ were also affected and reduces accordingly. The architecture with smallest RMSE and high $\mathrm{R}^{2}$ were selected as the best architecture. 
International Journal of Computer Applications Technology and Research

Volume 8-Issue 06, 229-268, 2019, ISSN:-2319-8656

Table 4.1: Architectures and statistical parameters for ANNs tested for $\mathrm{Ht}(\mathrm{cm})$

\begin{tabular}{|c|c|c|c|c|c|c|c|c|c|c|}
\hline \multicolumn{11}{|c|}{ ANNs for sampling data } \\
\hline $\begin{array}{l}\text { Network } \\
\text { Architecture }\end{array}$ & $1: 1: 1$ & $1: 2: 1$ & $1: 3: 1$ & $1: 4: 1$ & $1: 5: 1$ & $1: 6: 1$ & $1: 7: 1$ & $1: 8: 1$ & $1: 9: 1$ & $1: 10: 1$ \\
\hline $\begin{array}{l}\text { Min. } \\
\text { Performance } \\
\text { gradient }\end{array}$ & 0.00001 & 0.00001 & 0.00001 & 0.00001 & 0.00001 & 0.00001 & 0.00001 & 0.00001 & 0.00001 & 0.00001 \\
\hline $\begin{array}{l}\text { Max. Number of } \\
\text { epoch trained }\end{array}$ & 1000 & 1000 & 1000 & 1000 & 1000 & 1000 & 1000 & 1000 & 1000 & 1000 \\
\hline Learning rate $(\mathrm{n})$ & 0.01 & 0.01 & 0.01 & 0.01 & 0.01 & 0.01 & 0.01 & 0.01 & 0.01 & 0.01 \\
\hline Performance goal & 0.00 & 0.00 & 0.00 & 0.00 & 0.00 & 0.00 & 0.00 & 0.00 & 0.00 & 0.00 \\
\hline $\mathrm{R}^{2}$ & 0.9997 & 0.9988 & 0.9999 & 0.9996 & 0.9938 & 0.9996 & 0.9997 & 0.9957 & 0.9996 & 0.9997 \\
\hline RMSE & 2.3477 & 4.645 & $\mathbf{1 . 5 3 2 0}$ & 2.8564 & 10.2897 & 3.1259 & 2.7422 & 8.2836 & 2.882 & 2.5329 \\
\hline
\end{tabular}

Table 4.2: Architectures and statistical parameters for ANNs tested for G50 (cm)

\begin{tabular}{|c|c|c|c|c|c|c|c|c|c|c|}
\hline \multicolumn{11}{|c|}{ ANNs for sampling data } \\
\hline $\begin{array}{l}\text { Network } \\
\text { Architecture }\end{array}$ & $1: 1: 1$ & $1: 2: 1$ & $1: 3: 1$ & $1: 4: 1$ & $1: 5: 1$ & $1: 6: 1$ & $1: 7: 1$ & $1: 8: 1$ & $1: 9: 1$ & $1: 10: 1$ \\
\hline $\begin{array}{l}\text { Min. } \\
\text { Performance } \\
\text { gradient }\end{array}$ & 0.00001 & 0.00001 & 0.00001 & 0.00001 & 0.00001 & 0.00001 & 0.00001 & 0.00001 & 0.00001 & 0.00001 \\
\hline $\begin{array}{l}\text { Max. Number of } \\
\text { epoch trained }\end{array}$ & 1000 & 1000 & 1000 & 1000 & 1000 & 1000 & 1000 & 1000 & 1000 & 1000 \\
\hline Learning rate $(n)$ & 0.01 & 0.01 & 0.01 & 0.01 & 0.01 & 0.01 & 0.01 & 0.01 & 0.01 & 0.01 \\
\hline Performance goal & 0.00 & 0.00 & 0.00 & 0.00 & 0.00 & 0.00 & 0.00 & 0.00 & 0.00 & 0.00 \\
\hline $\mathrm{R}^{2}$ & 0.9995 & 0.9999 & 0.9991 & 0.9995 & 0.9996 & 0.9992 & 0.9997 & 0.9993 & 0.9767 & 0.9982 \\
\hline RMSE & 0.5469 & 0.2229 & 0.7997 & 0.6530 & 0.5525 & 0.6357 & 0.4178 & 0.6977 & 3.296 & 1.0237 \\
\hline
\end{tabular}


International Journal of Computer Applications Technology and Research

Volume 8-Issue 06, 229-268, 2019, ISSN:-2319-8656

Table 4.3: Architectures and statistical parameters for ANNs tested for NEL

\begin{tabular}{|c|c|c|c|c|c|c|c|c|c|c|}
\hline \multicolumn{11}{|c|}{ ANNs for sampling data } \\
\hline $\begin{array}{l}\text { Network } \\
\text { Architecture }\end{array}$ & $1: 1: 1$ & $1: 2: 1$ & $1: 3: 1$ & $1: 4: 1$ & $1: 5: 1$ & $1: 6: 1$ & $1: 7: 1$ & $1: 8: 1$ & $1: 9: 1$ & $1: 10: 1$ \\
\hline $\begin{array}{l}\text { Min. } \\
\text { Performance } \\
\text { gradient }\end{array}$ & 0.00001 & 0.00001 & 0.00001 & 0.00001 & 0.00001 & 0.00001 & 0.00001 & 0.00001 & 0.00001 & 0.00001 \\
\hline $\begin{array}{l}\text { Max. Number of } \\
\text { epoch trained }\end{array}$ & 1000 & 1000 & 1000 & 1000 & 1000 & 1000 & 1000 & 1000 & 1000 & 1000 \\
\hline Learning rate $(\eta)$ & 0.01 & 0.01 & 0.01 & 0.01 & 0.01 & 0.01 & 0.01 & 0.01 & 0.01 & 0.01 \\
\hline Performance goal & 0.00 & 0.00 & 0.00 & 0.00 & 0.00 & 0.00 & 0.00 & 0.00 & 0.00 & 0.00 \\
\hline $\mathrm{R}^{2}$ & 0.9993 & 0.9967 & 0.9997 & 0.9989 & 0.99895 & 0.99892 & 0.9991 & 0.99949 & 0.9983 & 0.8466 \\
\hline RMSE & 0.2857 & 0.6889 & 0.2202 & 0.3885 & 0.4051 & 0.4293 & 0.3561 & 0.29086 & 0.47941 & 4.1716 \\
\hline
\end{tabular}

Table 4.4: Architectures and statistical parameters for ANNs tested for LW (cm)

\begin{tabular}{|c|c|c|c|c|c|c|c|c|c|c|}
\hline \multicolumn{11}{|c|}{ ANNs for sampling data } \\
\hline $\begin{array}{l}\text { Network } \\
\text { Architecture }\end{array}$ & $1: 1: 1$ & $1: 2: 1$ & $1: 3: 1$ & $1: 4: 1$ & $1: 5: 1$ & $1: 6: 1$ & $1: 7: 1$ & $1: 8: 1$ & $1: 9: 1$ & $1: 10: 1$ \\
\hline $\begin{array}{l}\text { Min. } \\
\text { Performance } \\
\text { gradient }\end{array}$ & 0.00001 & 0.00001 & 0.00001 & 0.00001 & 0.00001 & 0.00001 & 0.00001 & 0.00001 & 0.00001 & 0.00001 \\
\hline $\begin{array}{l}\text { Max. Number of } \\
\text { epoch trained }\end{array}$ & 1000 & 1000 & 1000 & 1000 & 1000 & 1000 & 1000 & 1000 & 1000 & 1000 \\
\hline Learning rate $(\eta)$ & 0.01 & 0.01 & 0.01 & 0.01 & 0.01 & 0.01 & 0.01 & 0.01 & 0.01 & 0.01 \\
\hline Performance goal & 0.00 & 0.00 & 0.00 & 0.00 & 0.00 & 0.00 & 0.00 & 0.00 & 0.00 & 0.00 \\
\hline $\mathrm{R}^{2}$ & 0.99888 & 0.9995 & 0.99917 & 0.99866 & 0.99784 & 0.99915 & 0.99889 & 0.99845 & 0.99814 & 0.99691 \\
\hline RMSE & 0.61538 & 0.3656 & 0.56195 & 0.62776 & 0.81974 & 0.54533 & 0.57367 & 0.75485 & 0.66701 & 0.96756 \\
\hline
\end{tabular}


International Journal of Computer Applications Technology and Research

Volume 8-Issue 06, 229-268, 2019, ISSN:-2319-8656

Table 4.5: Architectures and statistical parameters for ANNs tested for LL (cm)

\begin{tabular}{|l|l|l|l|l|l|l|l|l|l|l|}
\hline \multicolumn{2}{|c|}{$\begin{array}{l}\text { ANNs for sampling data } \\
\text { Architecture }\end{array}$} & $1: 1: 1$ & $1: 2: 1$ & $1: 3: 1$ & $1: 4: 1$ & $1: 5: 1$ & $1: 6: 1$ & $1: 7: 1$ & $1: 8: 1$ & $1: 9: 1$ \\
\hline $\begin{array}{l}\text { Min. } \\
\begin{array}{l}\text { Performance } \\
\text { gradient }\end{array}\end{array}$ & 0.00001 & 0.00001 & 0.00001 & 0.00001 & 0.00001 & 0.00001 & 0.00001 & 0.00001 & 0.00001 & 0.00001 \\
\hline $\begin{array}{l}\text { Max. Number of } \\
\text { epoch trained }\end{array}$ & 1000 & 1000 & 1000 & 1000 & 1000 & 1000 & 1000 & 1000 & 1000 \\
\hline Learning rate (n) & 0.01 & 0.01 & 0.01 & 0.01 & 0.01 & 0.01 & 0.01 & 0.01 & 0.01 \\
\hline Performance goal & 0.00 & 0.00 & 0.00 & 0.00 & 0.00 & 0.00 & 0.00 & 0.00 & 0.00 \\
\hline $\mathrm{R}^{2}$ & 0.9986 & 0.9996 & 0.9998 & 0.9988 & 0.9998 & 0.9997 & 0.9997 & 0.9993 & $\mathbf{0 . 9 9 9 8}$ & 0.9995 \\
\hline RMSE & 2.1858 & 1.2897 & 1.1942 & 2.0256 & 1.36 & 1.2456 & 1.1886 & 1.5789 & $\mathbf{1 . 0 2 0 0}$ & 1.9438 \\
\hline
\end{tabular}

\subsection{Acceptance of Architecture}

After the network was fully trained (the network was trained over and again for 10 times) for the $\mathrm{Ht}, \mathrm{G} 50$, NEL, LW and LL, the figure 4.1 shows the regression plot graph for $\mathrm{Ht}$ and it was observed that the data were very close to the line of best fit. The dataset were divided to three, $70 \%$ of dataset for the training, $15 \%$ for validation and $15 \%$ for test. From figure 4.1 , the correlation coefficient of determination $\left(\mathrm{R}^{2}\right)$ for the overall is 0.9999 which (PFP) to the harvest time. At this point is when the plantain plant starts to bring out the NFB (number of fingers in the bunch) and NHB (number of hands in the bunch). The planting to flowering period is around 230 days for the dataset used for this network training. However, due to slight variation in data, there is a close range of values of data before the PFP to the PFP in the dataset (see Table 4.6 for the experimental dataset of a plant), which looks like the PFP starts before 200 days from the time series response graph but looking very closely in a magnified view, the PFP actually starts after 200 www.ijcat.com is a strong correlation value for $\mathrm{R}^{2}$. The corresponding correlation coefficient of determination $\left(\mathrm{R}^{2}\right)$ plot graph for G50, NEL, LW and LL are figure 4.7, figure 4.8 , figure 4.9 and figure 4.10 respectively.

Figure 4.2 shows the time series response graph for Ht. The height of the plants was increasing and becomes constant at planting to flowering period days. At 176 days (DAP: Days After Planting) from Table 4.6, it was observed that the range of values were very close to the PFP where the values become constant for all the parameters identified as the plantain growth parameters in this research. 
Comparing the time response graph of $\mathrm{Ht}$, G50, NEL,

LW and LL it was concluded that there is correlation from the time series response graph of these parameters. The values were constant at the same period. See Figure 4.3, Figure 4.4, Figure 4.5 and Figure 4.6 for the time series response graph for G50, NEL, LW and LL respectively.

Table 4.6: dataset of a plant used for training.

\begin{tabular}{|l|l|l|l|l|l|l|l|l|}
\hline DAP & Ht & G50 & NEL & LW & LL & BW & NHB & NFB \\
\hline 35 & 28 & 6.5 & 2 & 18 & 35 & 0 & 0 & 0 \\
\hline 49 & 38 & 9.5 & 4.8 & 24.5 & 47 & 0 & 0 & 0 \\
\hline 63 & 50 & 12 & 7.2 & 31 & 55.8 & 0 & 0 & 0 \\
\hline 77 & 77.2 & 18 & 10.2 & 39.2 & 77 & 0 & 0 & 0 \\
\hline 92 & 107 & 27.9 & 13.4 & 49 & 93.5 & 0 & 0 & 0 \\
\hline 105 & 141 & 29.3 & 16 & 55.5 & 120.2 & 0 & 0 & 0 \\
\hline 121 & 172 & 38.5 & 19 & 61.2 & 149.7 & 0 & 0 & 0 \\
\hline 133 & 196 & 47 & 21 & 72 & 166 & 0 & 0 & 0 \\
\hline 148 & 218 & 49.8 & 23.2 & 71.5 & 184 & 0 & 0 & 0 \\
\hline 162 & 257 & 58.8 & 25.4 & 78 & 201 & 0 & 0 & 0 \\
\hline 176 & 285 & 67 & 27.4 & 74 & 214 & 0 & 0 & 0 \\
\hline 190 & 295 & 70 & 31.8 & 74.6 & 217 & 0 & 0 & 0 \\
\hline 203 & 322 & 73 & 31.2 & 74.6 & 217 & 0 & 0 & 0 \\
\hline 217 & 339 & 68 & 32 & 74.6 & 217 & 0 & 0 & 0 \\
\hline 232 & 325 & 70 & 32 & 74.6 & 217 & 0 & 7 & 99 \\
\hline 246 & 325 & 70 & 32 & 74.6 & 217 & 0 & 7 & 99 \\
\hline 260 & 325 & 70 & 32 & 74.6 & 217 & 0 & 7 & 99 \\
\hline 273 & 325 & 70 & 32 & 74.6 & 217 & 0 & 7 & 99 \\
\hline 288 & 325 & 70 & 32 & 74.6 & 217 & 0 & 7 & 99 \\
\hline 302 & 325 & 70 & 32 & 74.6 & 217 & 0 & 7 & 99 \\
\hline 317 & 325 & 70 & 32 & 74.6 & 217 & 0 & 7 & 99 \\
\hline 321 & 325 & 70 & 32 & 74.6 & 217 & 20 & 7 & 99 \\
\hline
\end{tabular}



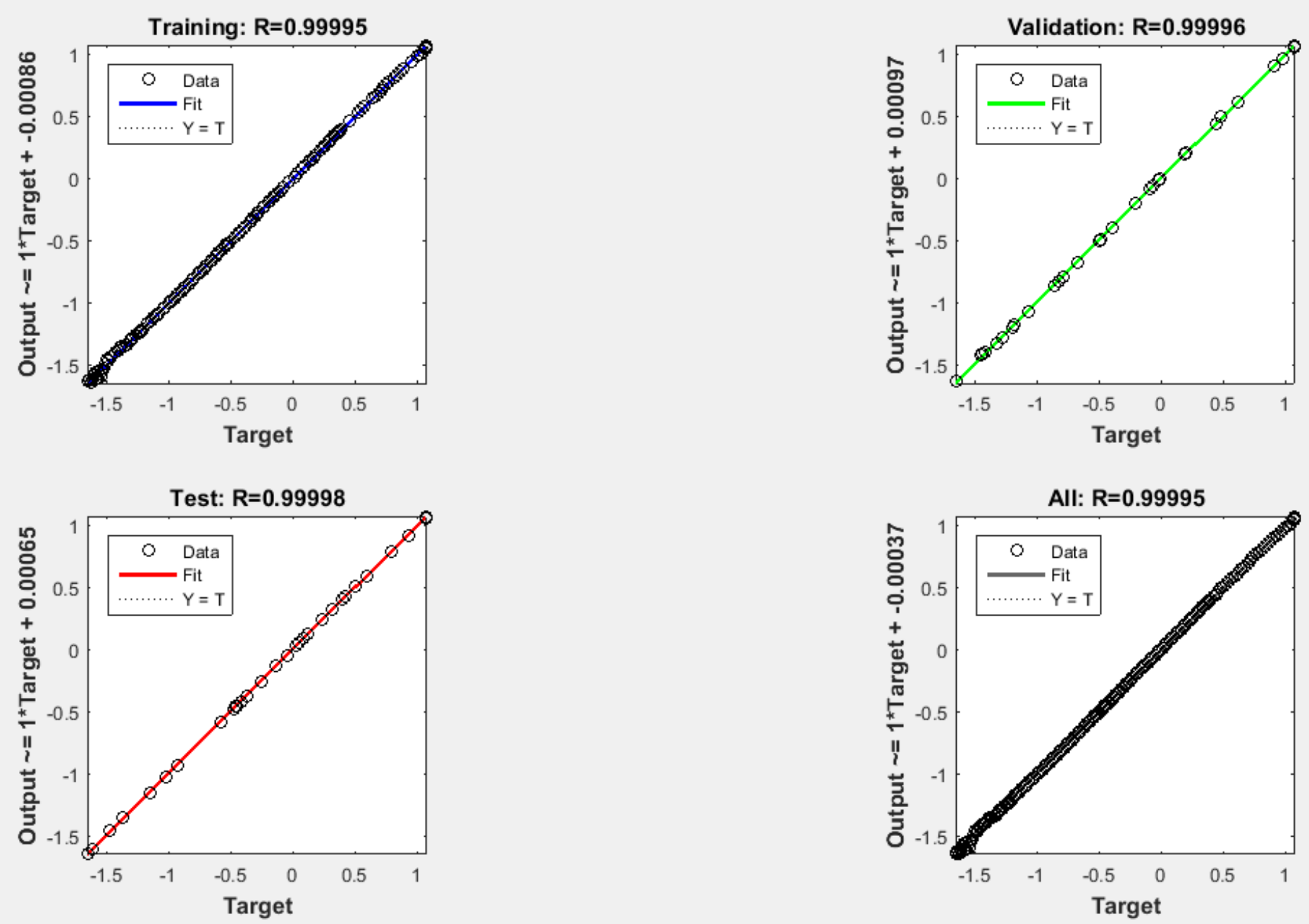

Figure 4.1: The regression plot for $\mathrm{Ht}$ 
International Journal of Computer Applications Technology and Research

Volume 8-Issue 06, 229-268, 2019, ISSN:-2319-8656
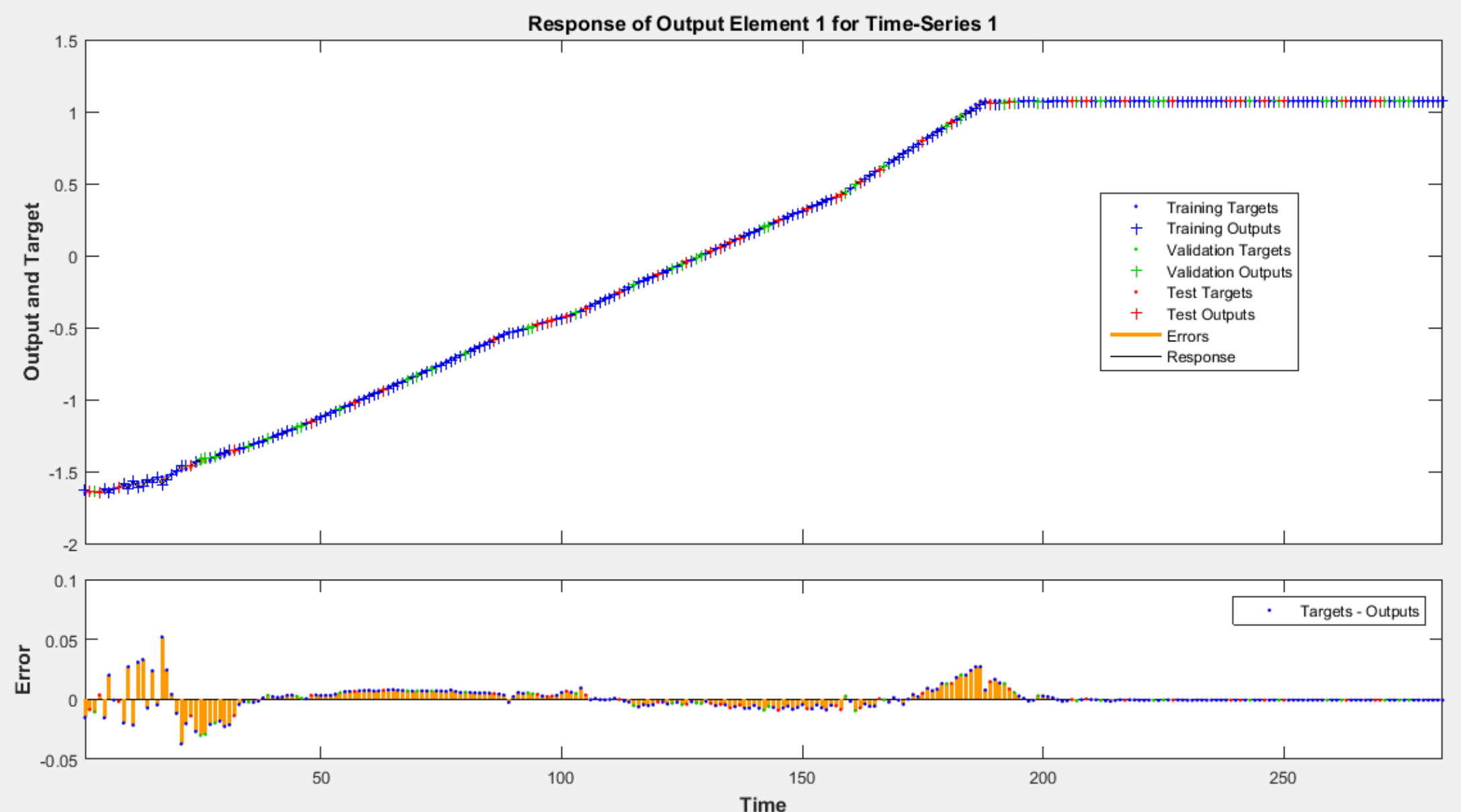

Figure 4.2: time series response graph for $\mathrm{Ht}$ 
International Journal of Computer Applications Technology and Research

Volume 8-Issue 06, 229-268, 2019, ISSN:-2319-8656

\section{Response of Output Element 1 for Time-Series 1}
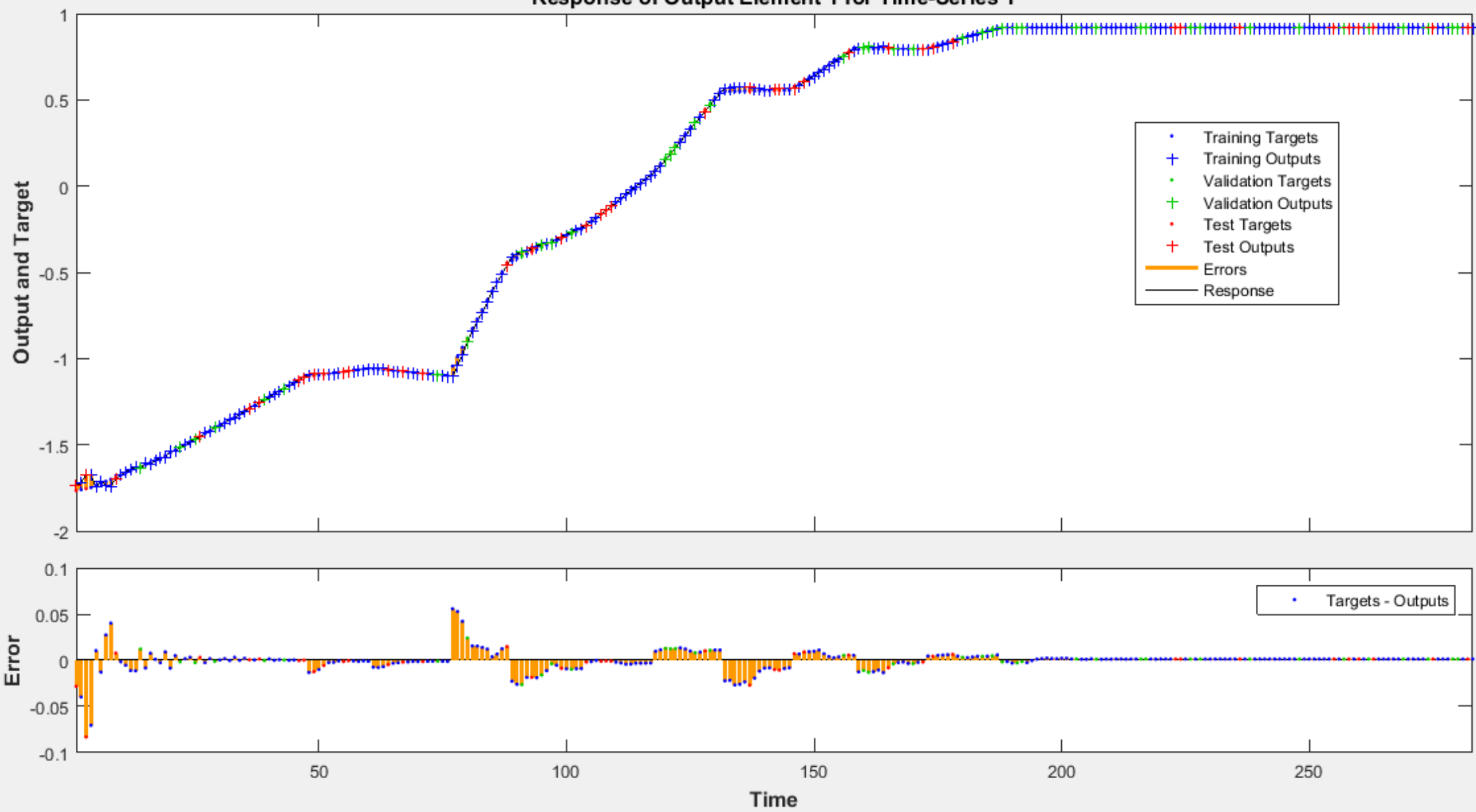

Figure 4.3: time series response graph for G50 
International Journal of Computer Applications Technology and Research

Volume 8-Issue 06, 229-268, 2019, ISSN:-2319-8656

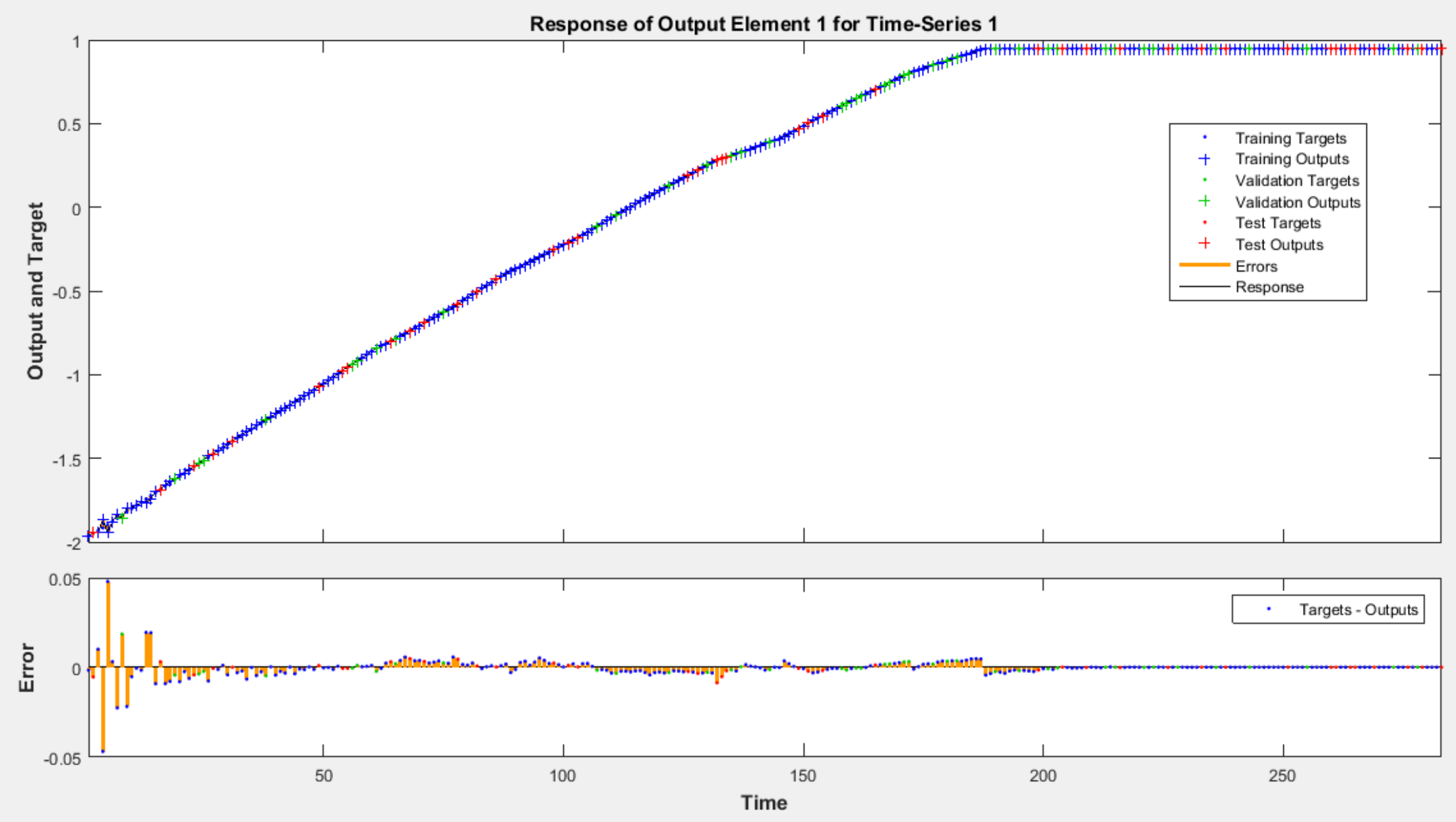

Figure 4.4: time series response graph for NEL 
International Journal of Computer Applications Technology and Research

Volume 8-Issue 06, 229-268, 2019, ISSN:-2319-8656
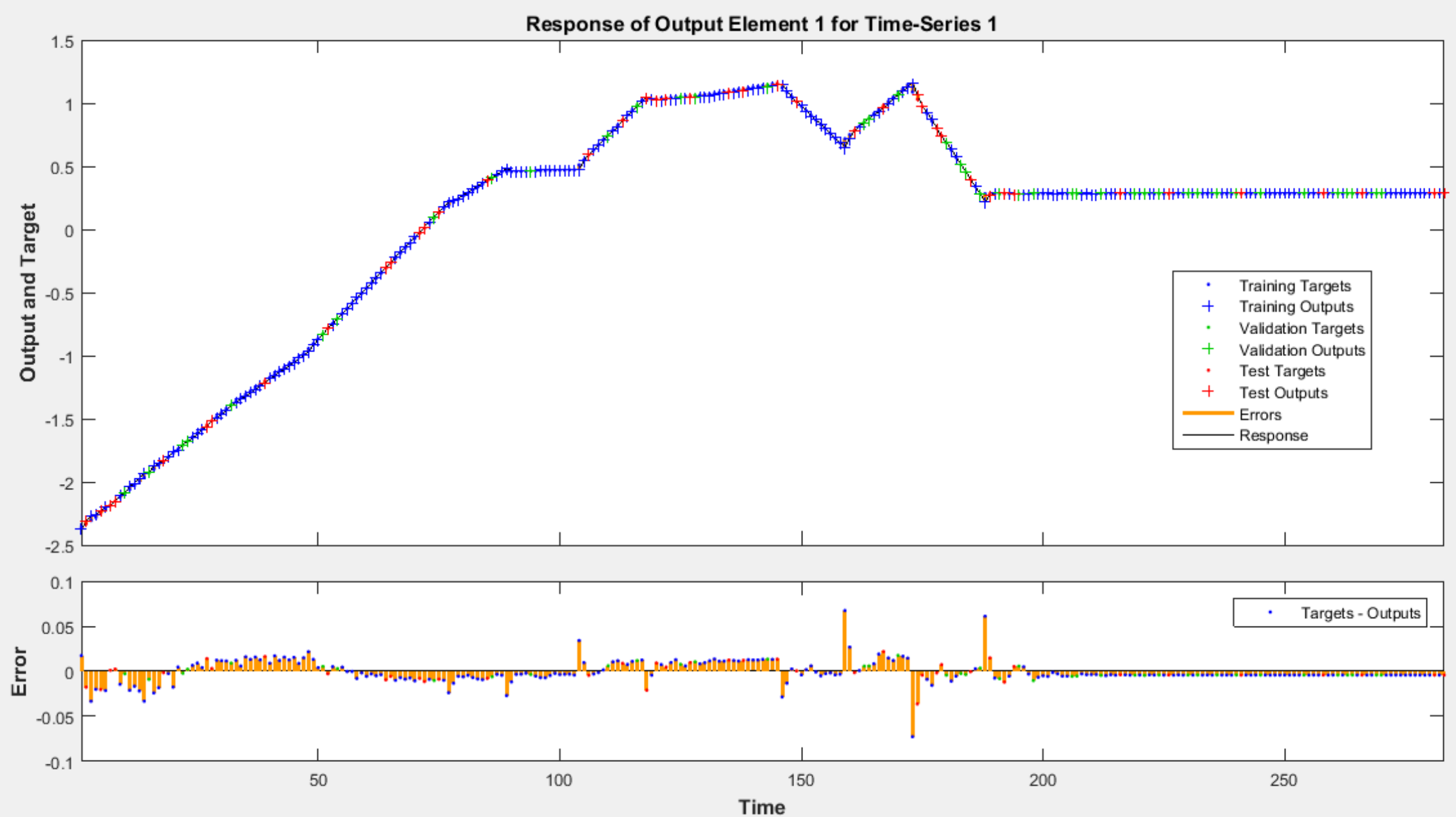

Figure 4.5: time series response graph for LW 
International Journal of Computer Applications Technology and Research

Volume 8-Issue 06, 229-268, 2019, ISSN:-2319-8656
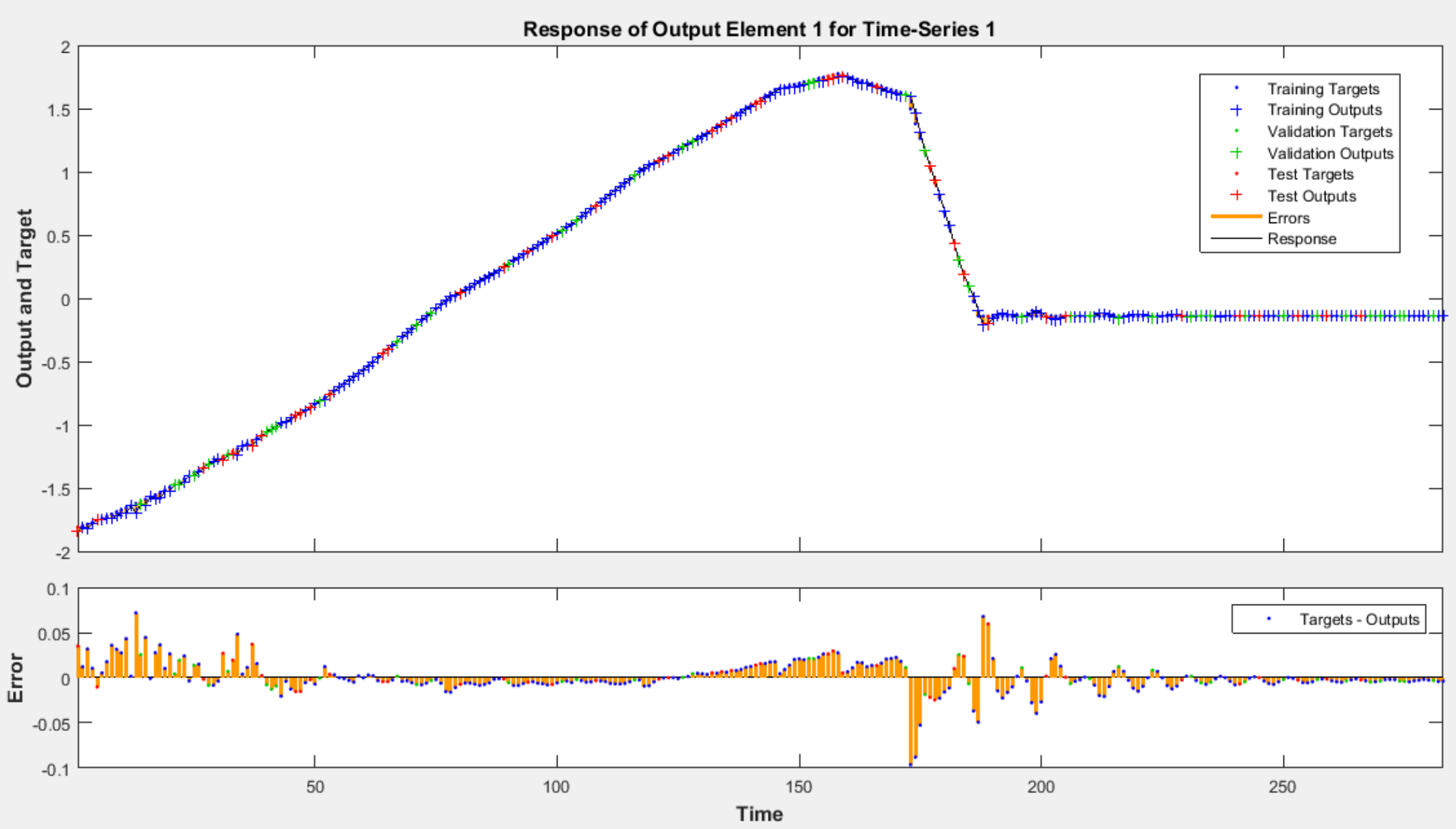

Figure 4.6: time series response graph for $\mathrm{LL}$ 

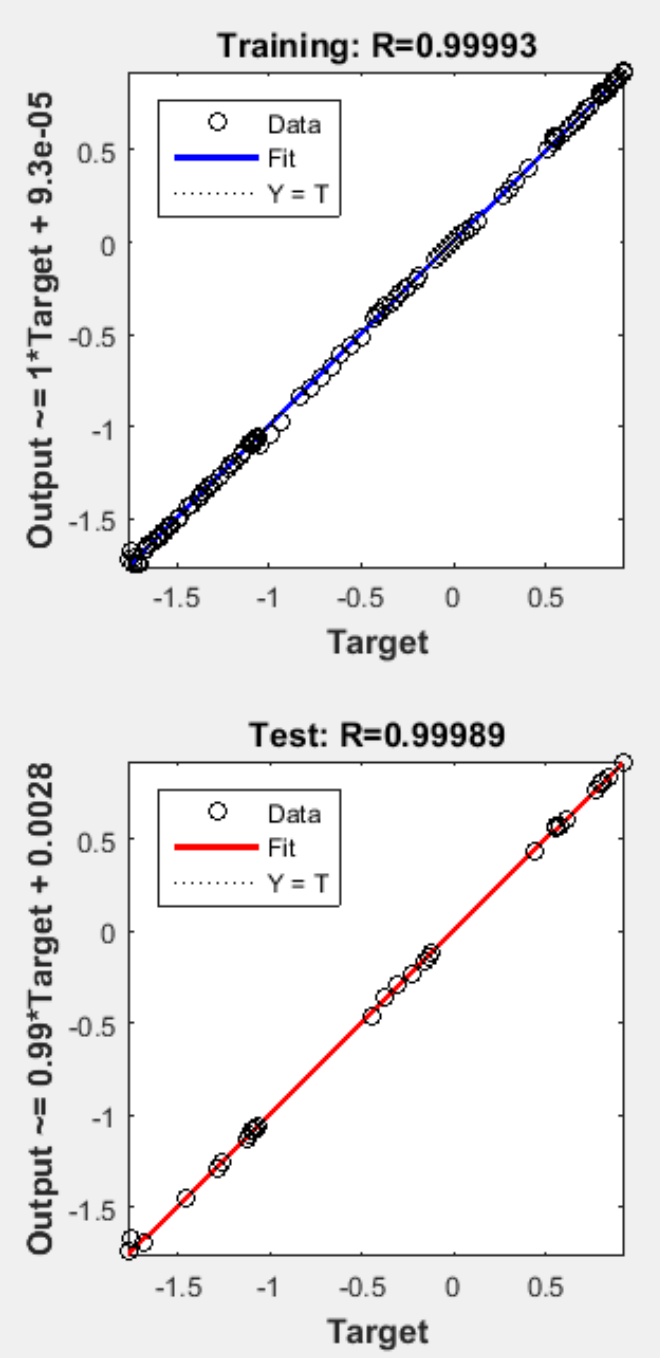
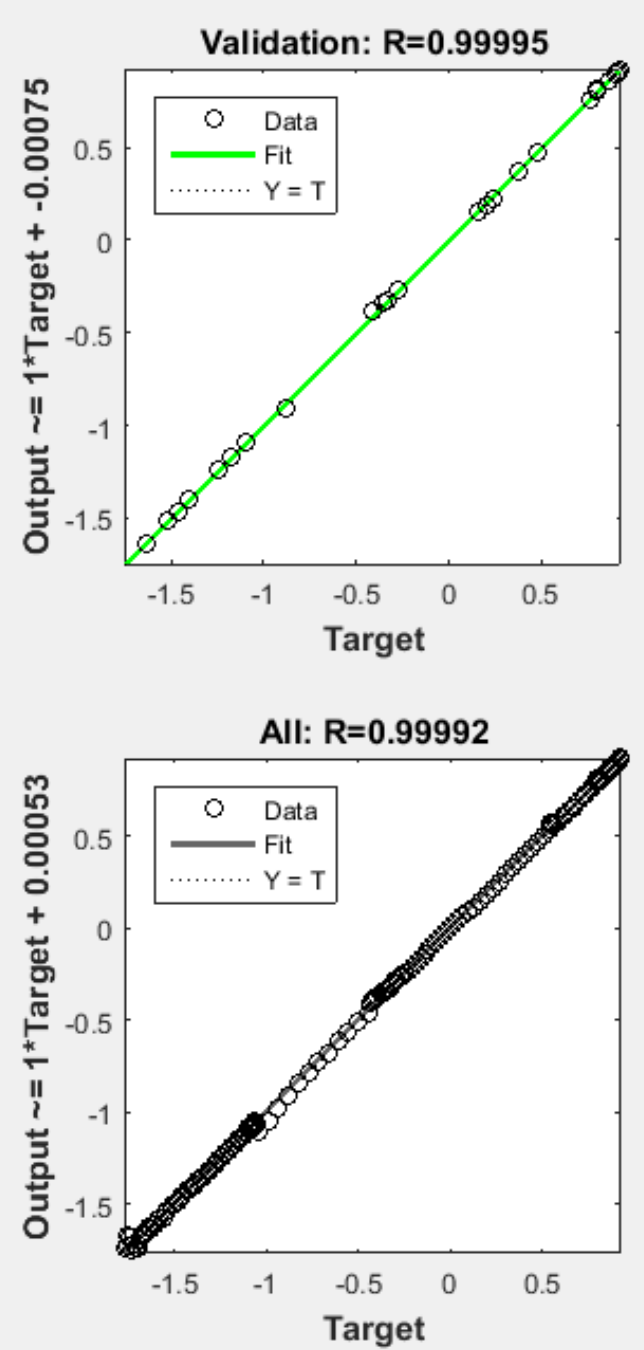

Figure 4.7: The regression plot for G50 

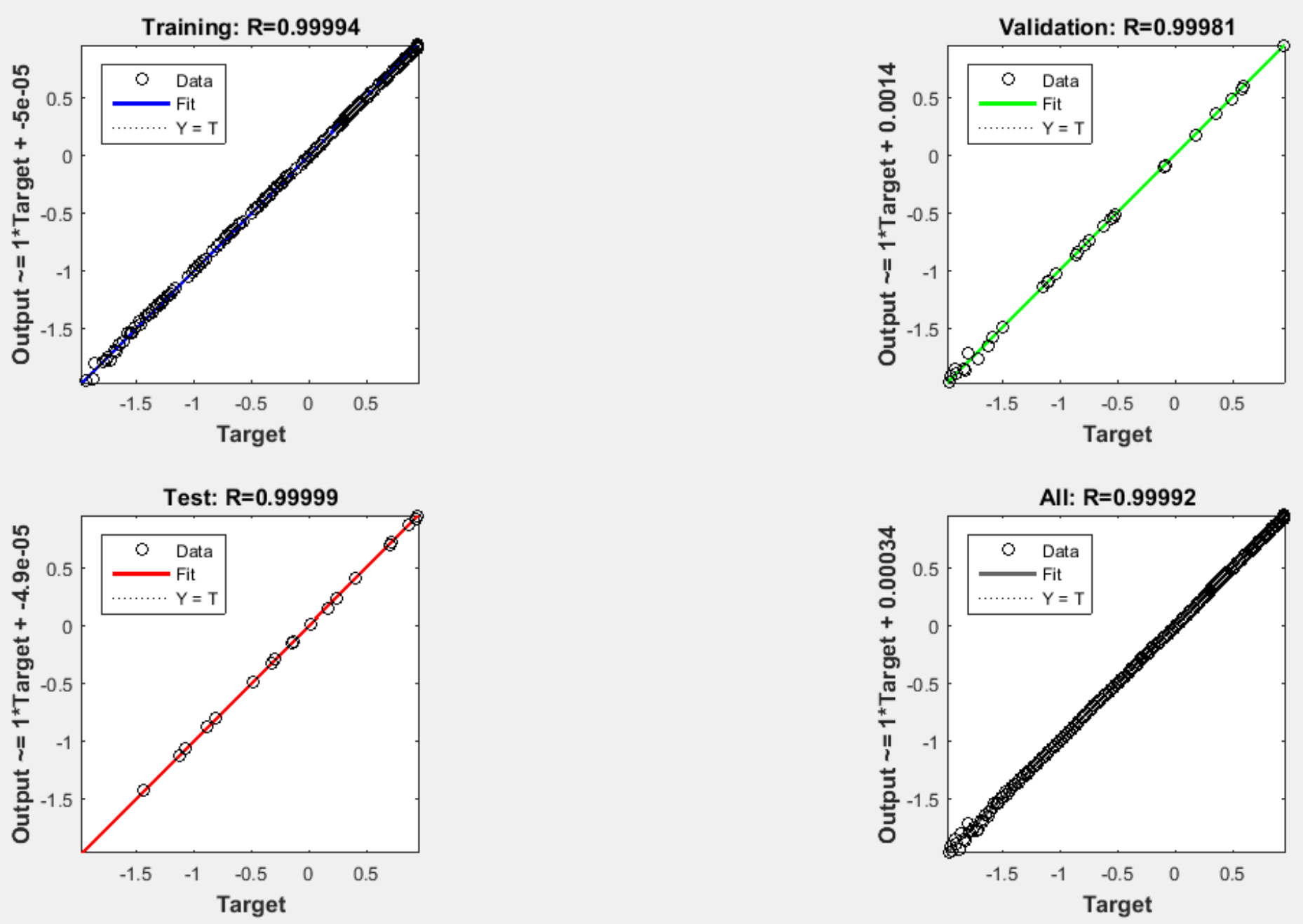

Figure 4.8: The regression plot for NEL 

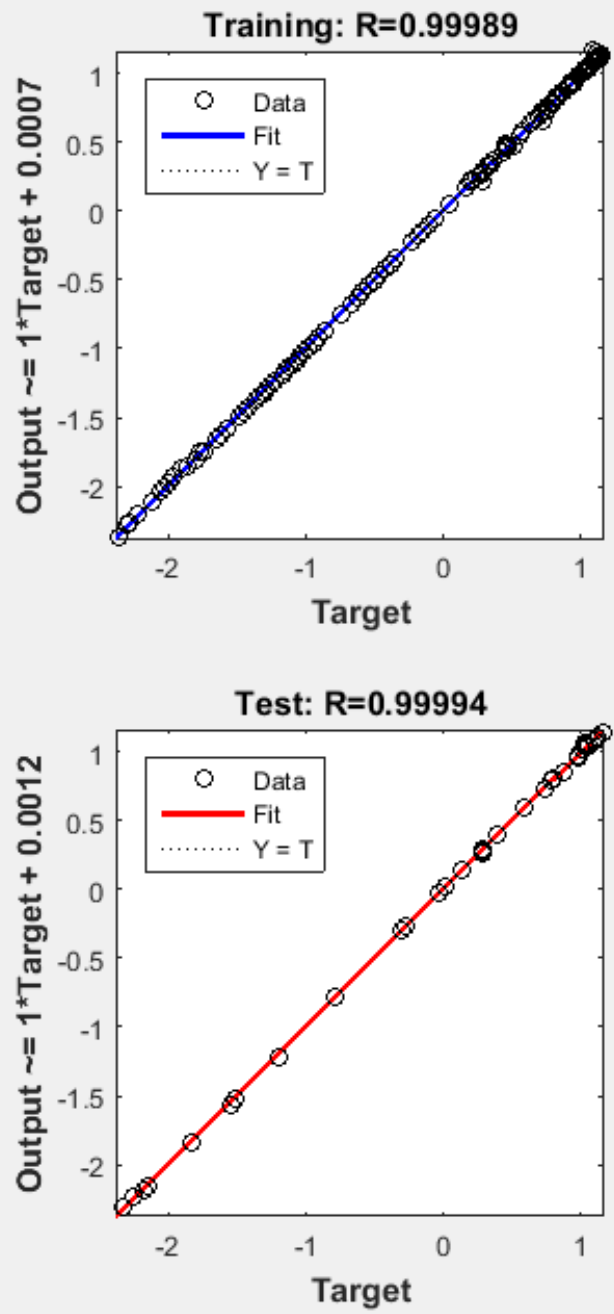
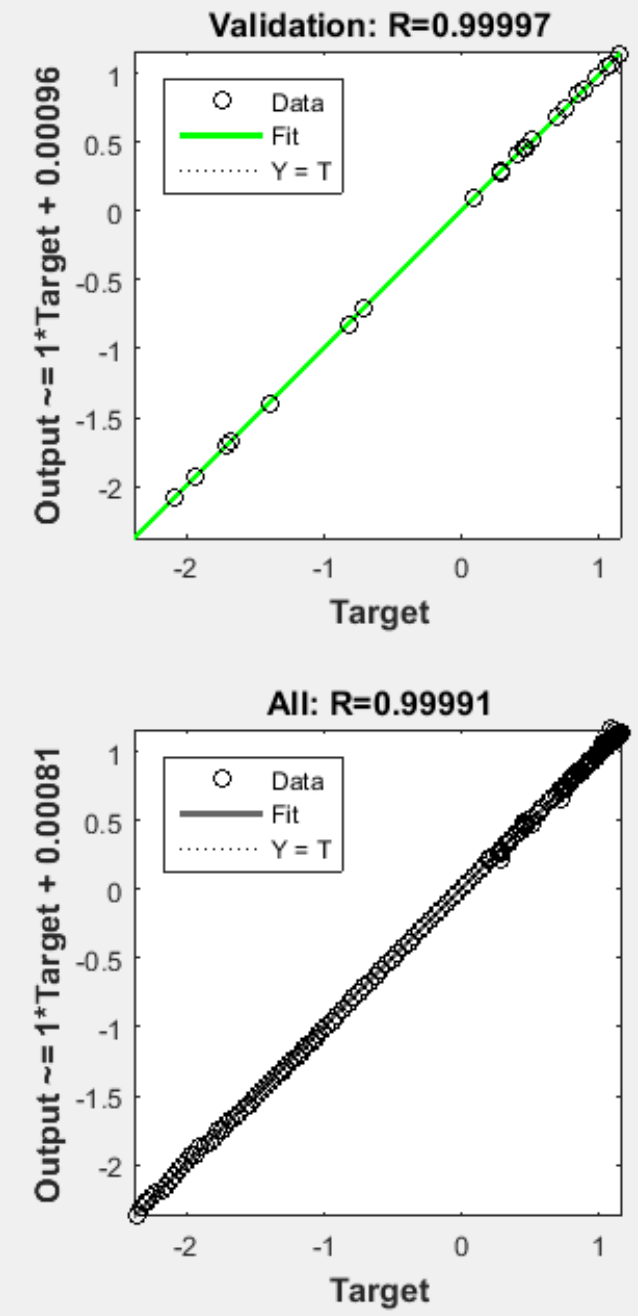

Figure 4.9: The regression plot for LW 

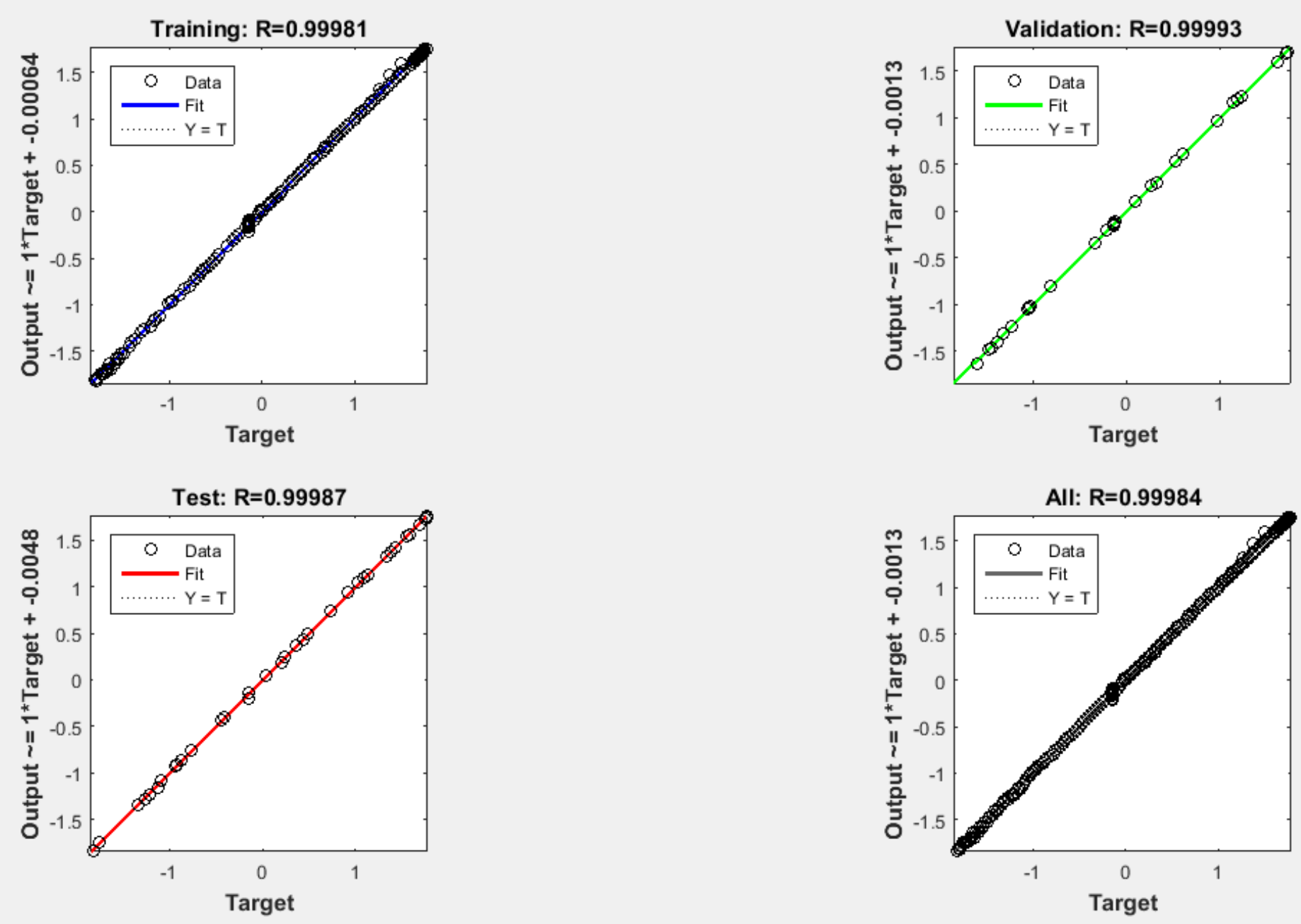

Figure 4.10: The regression plot for LL 


\subsection{Predicting Values}

The network results of $\mathrm{Ht}, \mathrm{G} 50$, NEL, LW and LL, are used to train the networks of BW, NHB and NFB. Their corresponding coefficients of determination $\left(\mathrm{R}^{2}\right)$ and the Root Mean Squared Error (RMSE) were recorded to determine the acceptance of each network architecture. See Table 4.7, Table 4.8 and Table 4.9 for the architectures and statistical parameters for ANNs tested for BW, NHB and NFB respectively and the coefficients of determination $\left(\mathrm{R}^{2}\right)$ plot graph for each of these networks, see figure 4.11, figure 4.12 and figure 4.13 respectively. There are no enough data for the neural network to train each of BW, NHB and NFB networks. However, their training results can still be accepted base on the $\mathrm{R}^{2}$ values and the RMSE values. The architecture for BW network is 1:15:1, the architecture for NHB network is 1:25:1 and the architecture for NFB network is 1:10:1.

After all these parameters have been trained, the network predicted into future the values of plantain height, the girth at $50 \mathrm{~cm}$ above the soil level, number of emitted leaves, leaf width, leaf length, bunch weight of plantain, number of hands in the plantain bunch and number of fingers in the plantain bunch given the known values (from the plantain sucker, an experimental value) of Ht, G50, NEL, LW and LL to the network. Data (see Table 4.10) was supplied from the dataset of a plant ('Plantain-Optim' dataset) that has not been shown to the network, the result of the network was given in Table 4.11.

Table 4.7: Architectures and statistical parameters for ANNs tested for BW (Kg)

\begin{tabular}{|c|c|c|c|c|c|}
\hline Network Architecture & $1: 5: 1$ & $1: 10: 1$ & $1: 15: 1$ & $1: 20: 1$ & $1: 25: 1$ \\
\hline Min. Performance gradient & 0.0000001 & 0.0000001 & 0.0000001 & 0.0000001 & 0.0000001 \\
\hline Max. Number of epoch trained & 1000 & 1000 & 1000 & 1000 & 1000 \\
\hline Learning rate $(\eta)$ & 0.01 & 0.01 & 0.01 & 0.01 & 0.01 \\
\hline Performance goal & 0.00 & 0.00 & 0.00 & 0.00 & 0.00 \\
\hline $\mathrm{R}^{2}$ & 0.71907 & 0.8274 & 0.8294 & 0.66319 & 0.76053 \\
\hline RMSE & 2.422 & 1.9424 & 1.9391 & 2.6717 & 2.4599 \\
\hline
\end{tabular}


International Journal of Computer Applications Technology and Research

Volume 8-Issue 06, 229-268, 2019, ISSN:-2319-8656

Table 4.8: Architectures and statistical parameters for ANNs tested for NHB

\begin{tabular}{|c|c|c|c|c|c|}
\hline Network Architecture & $1: 5: 1$ & $1: 10: 1$ & $1: 15: 1$ & $1: 20: 1$ & $1: 25: 1$ \\
\hline Min. Performance gradient & 0.0000001 & 0.0000001 & 0.0000001 & 0.0000001 & 0.0000001 \\
\hline Max. Number of epoch trained & 1000 & 1000 & 1000 & 1000 & 1000 \\
\hline Learning rate $(n)$ & 0.01 & 0.01 & 0.01 & 0.01 & 0.01 \\
\hline Performance goal & 0.00 & 0.00 & 0.00 & 0.00 & 0.00 \\
\hline $\mathrm{R}^{2}$ & 0.77781 & 0.9224 & 0.89317 & 0.83905 & 0.9448 \\
\hline RMSE & 0.3730 & 0.2496 & 0.2902 & 0.3330 & 0.2134 \\
\hline
\end{tabular}

Table 4.9: Architectures and statistical parameters for ANNs tested for NFB

\begin{tabular}{|c|c|c|c|c|c|}
\hline Network Architecture & $1: 5: 1$ & $1: 10: 1$ & $1: 15: 1$ & $1: 20: 1$ & $1: 25: 1$ \\
\hline Min. Performance gradient & 0.0000001 & 0.0000001 & 0.0000001 & 0.0000001 & 0.0000001 \\
\hline $\begin{array}{l}\text { Max. Number of epoch } \\
\text { trained }\end{array}$ & 1000 & 1000 & 1000 & 1000 & 1000 \\
\hline Learning rate $(\eta)$ & 0.01 & 0.01 & 0.01 & 0.01 & 0.01 \\
\hline Performance goal & 0.00 & 0.00 & 0.00 & 0.00 & 0.00 \\
\hline $\mathrm{R}^{2}$ & 0.88915 & 0.92419 & 0.91731 & 0.93217 & 0.89562 \\
\hline RMSE & 13.4241 & 11.2626 & 12.8368 & 10.7898 & 13.1776 \\
\hline
\end{tabular}



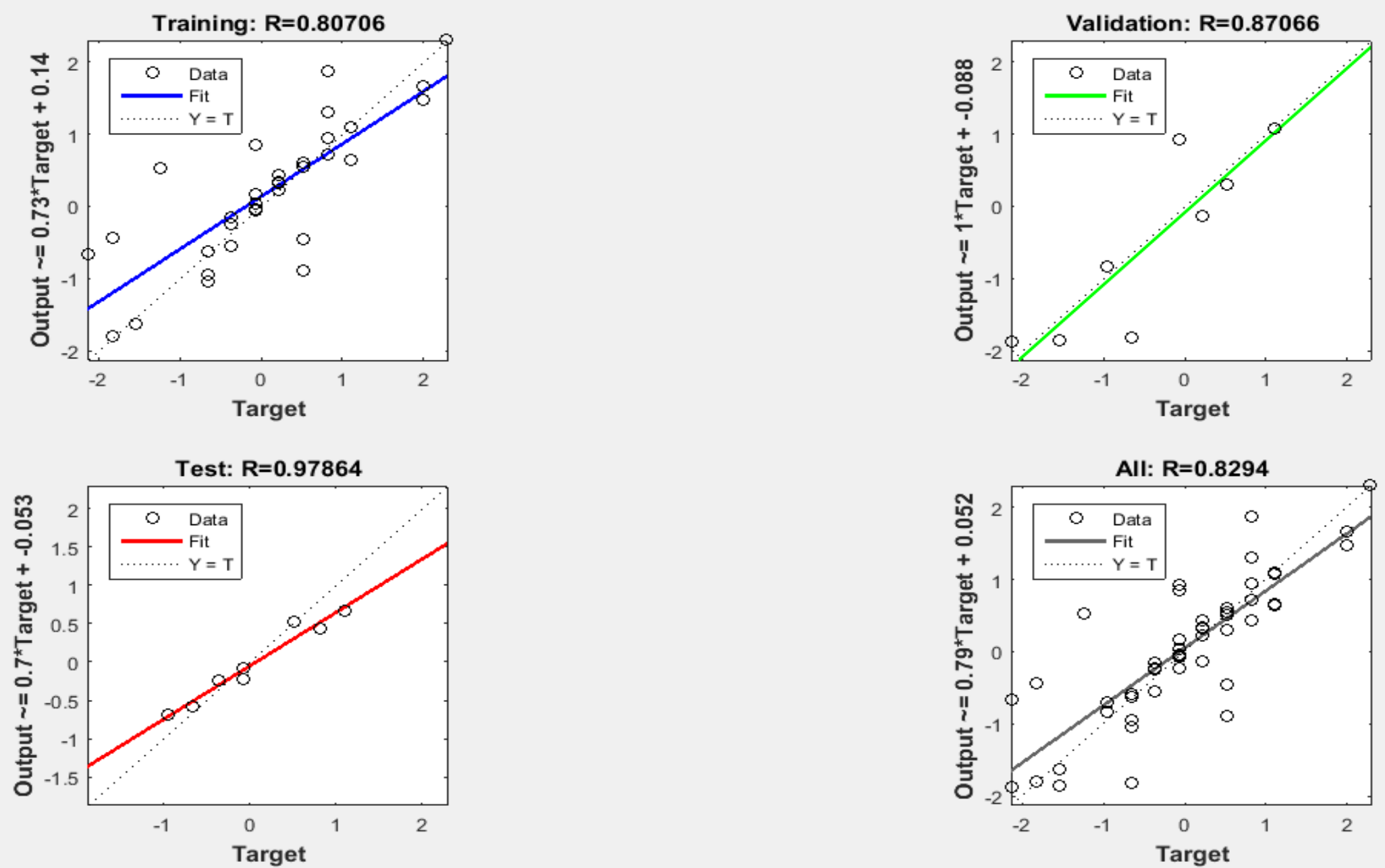

Figure 4.11: The regression plot for BW $(\mathrm{Kg})$ 

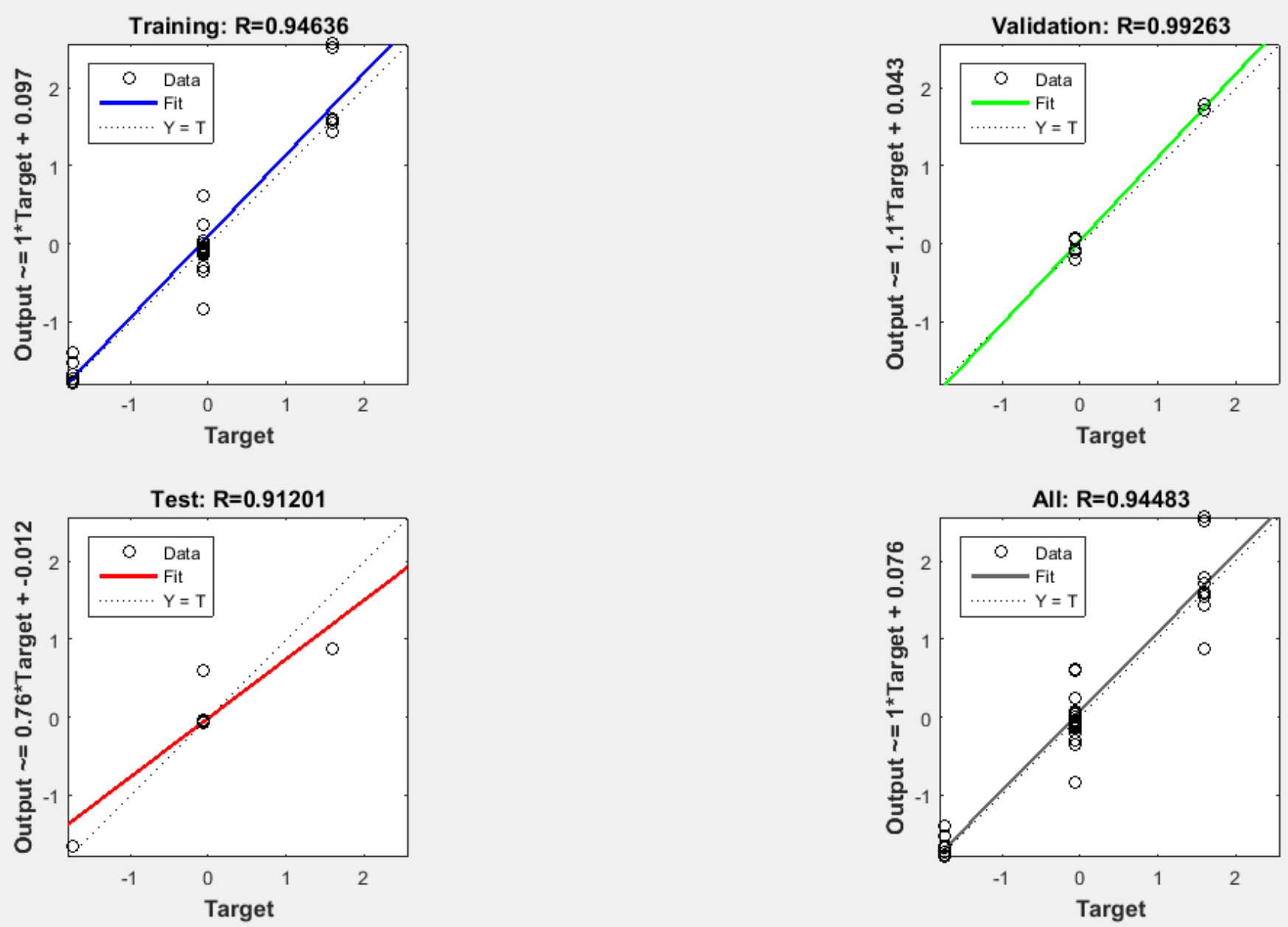

Figure 4.12: The regression plot for NHB 

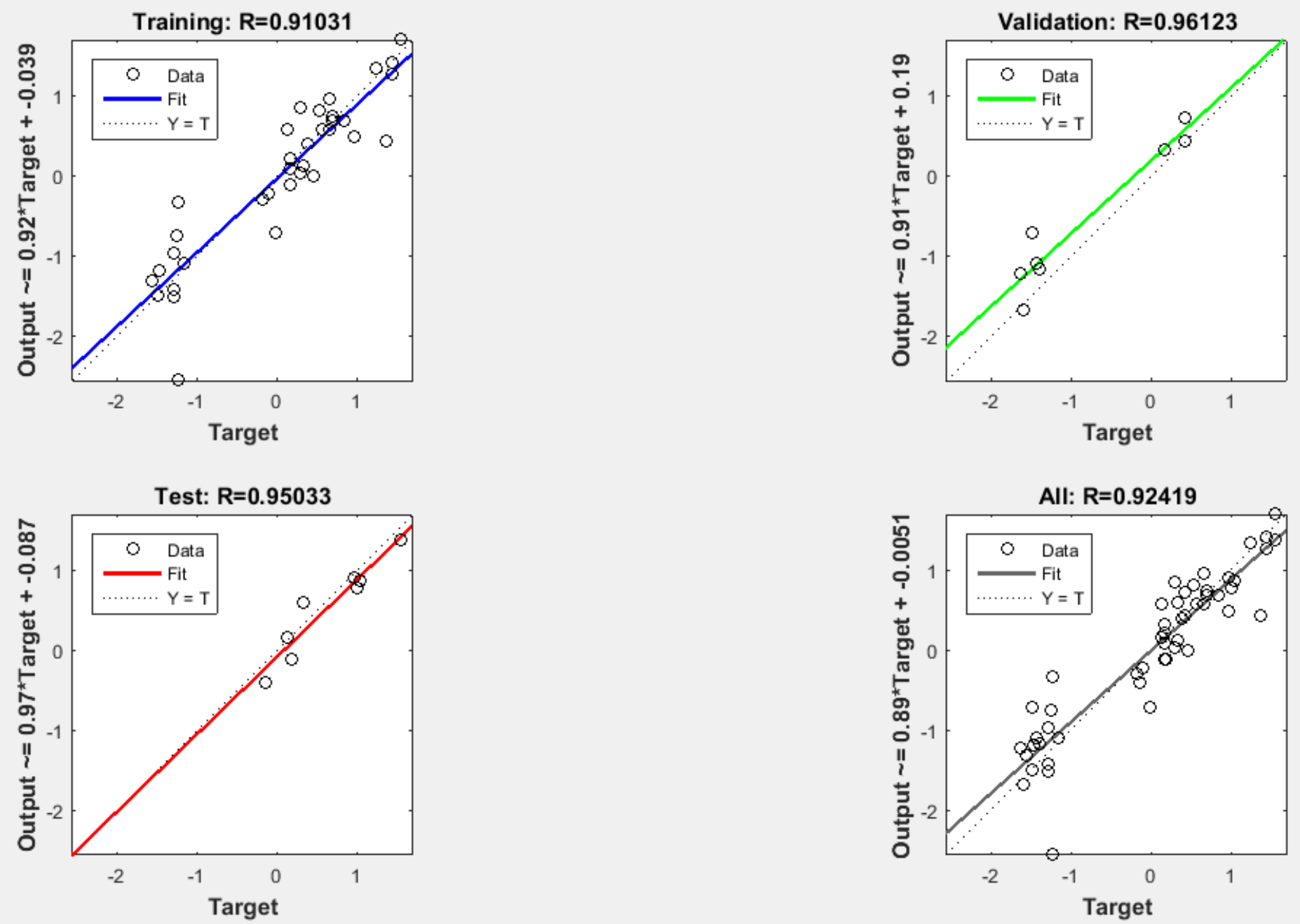

Figure 4.13: The regression plot for NFB 
Table 4.10: Dataset of a plant not shown to the network during training

\begin{tabular}{|c|c|c|c|c|c|c|c|c|}
\hline DAP & $\mathrm{Ht}$ & G50 & NEL & LW & $\mathrm{LL}$ & BW & $\mathrm{NHB}$ & NFB \\
\hline 35 & 22 & 8 & 2 & 25 & 37 & 0 & 0 & 0 \\
\hline 49 & 35 & 10 & 5 & 28.6 & 50.2 & 0 & 0 & 0 \\
\hline 63 & 52 & 14 & 8 & 32 & 63 & 0 & 0 & 0 \\
\hline 77 & 76.4 & 20.8 & 11 & 42.4 & 81 & 0 & 0 & 0 \\
\hline 92 & 108 & 28 & 14.6 & 56 & 106 & 0 & 0 & 0 \\
\hline 105 & 134 & 29.2 & 17.2 & 50.5 & 117 & 0 & 0 & 0 \\
\hline 121 & 162 & 37.4 & 20 & 70 & 146 & 0 & 0 & 0 \\
\hline 133 & 195 & 43.6 & 22.6 & 73 & 168 & 0 & 0 & 0 \\
\hline 148 & 220 & 53 & 25 & 77.3 & 189 & 0 & 0 & 0 \\
\hline 162 & 254 & 60.4 & 27.4 & 82 & 199 & 0 & 0 & 0 \\
\hline 176 & 277 & 67.3 & 30 & 89 & 225 & 0 & 0 & 0 \\
\hline 190 & 303 & 71 & 32 & 88 & 227.7 & 0 & 0 & 0 \\
\hline 203 & 341 & 77 & 34.2 & 91 & 270 & 0 & 0 & 0 \\
\hline 217 & 390 & 73 & 36.4 & 81 & 234 & 0 & 0 & 0 \\
\hline 232 & 400 & 74 & 37 & 81 & 234 & 0 & 0 & 0 \\
\hline 246 & 400 & 74 & 37 & 81 & 234 & 0 & 7 & 80 \\
\hline 260 & 400 & 74 & 37 & 81 & 234 & 0 & 7 & 80 \\
\hline 273 & 400 & 74 & 37 & 81 & 234 & 0 & 7 & 80 \\
\hline 288 & 400 & 74 & 37 & 81 & 234 & 0 & 7 & 80 \\
\hline 302 & 400 & 74 & 37 & 81 & 234 & 0 & 7 & 80 \\
\hline 317 & 400 & 74 & 37 & 81 & 234 & 0 & 7 & 80 \\
\hline 318 & 400 & 74 & 37 & 81 & 234 & 26 & 7 & 80 \\
\hline
\end{tabular}


Table 4.11: Elman Network Results when DAP was 121 at current and 318 for future values

\begin{tabular}{|l|l|}
\hline \multicolumn{2}{|c|}{ Known values to network } \\
\hline Enter the number of days to future & 318 \\
\hline Current number of days & 121 \\
\hline Enter current value of Ht & 162 \\
\hline Enter current value of G50 & 37.4 \\
\hline Enter current value of NEL & 20 \\
\hline Enter current value of LW & 70 \\
\hline Enter current value of LL & 146 \\
\hline & \\
\hline Predicted value of Ht to future & 476.0604 \\
\hline Predicted value of G50 to future & 31.2002 \\
\hline Predicted value of NEL to future & 5.4523 \\
\hline Predicted value of LW to future & 129.863 \\
\hline Predicted value of LL to future & 296.9355 \\
\hline Predicted value of BW to future & 49.7751 \\
\hline Predicted value of NHB to future & 7.0331 \\
\hline Predicted value of NFB to future & 123.4779 \\
\hline
\end{tabular}

\subsection{Evaluation of Predicted Values}

The known values supplied to the network for prediction at current values was at 121 days (DAP) and the number of days to future was the harvest period at 318 (see Table 4.10). The variation in the predicted values and the experimented are summarized in Table 4.12. These variations can be explained from the table of architectures and statistical parameters for ANNs tested for each of the parameters and secondly, the sample data size used for www.ijcat.com the training is too small for training neural network, as a result, the prediction can be very more accurate with enough dataset available for the network to train. Also the dataset used has five varieties and each variety of plantain have different variations under the same planting conditions on the farm field.

For Ht, the RMSE is 1.532, though this value is high but can still be accepted since the metric unit is in $\mathrm{cm}$ and the variation that occurred in the predicted 
value is still in a margin of acceptance since we are predicting the growth of plant and all plants cannot have the same height under the same planting condition.

For G50, the RMSE is 0.2229 , the result of the network can still be accepted for this parameter since the margin difference in $\mathrm{cm}$ is not much for a plant. However, for NEL, the margin error is too much. If network could be trained

\begin{tabular}{|l|l|l|l|}
\hline \multicolumn{1}{|c|}{ Parameters } & $\begin{array}{c}\text { Experimented } \\
\text { values }\end{array}$ & \multicolumn{1}{|c|}{$\begin{array}{c}\text { Predicted } \\
\text { values }\end{array}$} & Difference \\
\hline Ht $(\mathrm{cm})$ & 400 & 476.0604 & 76.0604 \\
\hline G50 (cm) & 74 & 31.2002 & 42.7998 \\
\hline NEL (unit) & 37 & 5.4523 & 31 \\
\hline LW (cm) & 81 & 129.863 & 48.863 \\
\hline LL (cm) & 234 & 296.9355 & 62.9355 \\
\hline BW (Kg) & 26 & 49.7751 & 23.7751 \\
\hline NHB (unit) & 7 & 7.0331 & 0.0331 \\
\hline NFB (unit) & 80 & 123.4779 & 43.4779 \\
\hline
\end{tabular}

\subsection{Time-delay Neural Network compared to Elman Networks}

Further research to prove the acceptance of result of the Elman neural network developed, it was observed that Elman network output saturates after specific number of steps. When it was tested on another input, when the known values supplied to the network for prediction at current values was at 63 days (DAP) and the number of days to future was at 148 days, a future day that is not the harvest period from Table 4.10. The result of the network was not really differ, no significant change between the results generated by Elman network with Table 4.11. Table 4.13 is the result outputted by the Elman network when current values of parameters were at 63 days and the expected future values were at 148 days. The values of BW, NHB, and NFB are NIL because 148 days was not the harvest period. These values only www.ijcat.com with more dataset, it would be more acceptable. LW and LL can be more accurate if network has enough data for training and NHB is acceptable because the difference is not eligible. BW, NHB and NFB were trained from the values obtained from Ht, G50, NEL, LW and LL networks. Too much divergence in the values of these 5 parameters would greatly affect the outputs of BW, NHB and NFB. appear in real life when the harvest period is close, which is after the flowering period. The flowering period can be observed from the time series response graphs where the values become constant.

Elman neural networks use simplified derivative calculations (using staticderiv - Static derivative function, which ignores delayed connections) at the expense of less reliable learning. This function calculates derivatives using the chain rule from the networks performance or outputs back to its inputs. For time series data and dynamic networks this function ignores the delay connections resulting in an approximation (which may be 
good or not) of the actual derivative which is not recommended for dynamic networks

Further to this approach, Time-delay neural network was introduced as an option to Elman neural network and it was observed that the network result

Table 4.13: Elman Network Results when DAP was 63 at current and 148 at future values

\begin{tabular}{|c|c|}
\hline \multicolumn{2}{|c|}{ Known values to network } \\
\hline Enter the number of days to future & 148 \\
\hline Current number of days & 63 \\
\hline Enter current value of $\mathrm{Ht}$ & 52 \\
\hline Enter current value of G50 & 14 \\
\hline Enter current value of NEL & 8 \\
\hline Enter current value of LW & 32 \\
\hline Enter current value of LL & 63 \\
\hline \multicolumn{2}{|c|}{ The network outputs for 148 days } \\
\hline Predicted value of $\mathrm{Ht}$ to future & 476.0604 \\
\hline Predicted value of G50 to future & 31.2002 \\
\hline Predicted value of NEL to future & 5.4523 \\
\hline Predicted value of LW to future & 129.863 \\
\hline Predicted value of LL to future & 37.4404 \\
\hline Predicted value of $\mathrm{BW}$ to future & NIL \\
\hline Predicted value of NHB to future & NIL \\
\hline Predicted value of NFB to future & NIL \\
\hline
\end{tabular}

Table 4.14: Time-delay neural network Results when DAP was 63 at current and 148 at future values

\begin{tabular}{|l|l|}
\hline \multicolumn{2}{|c|}{ Known values to network } \\
\hline Enter the number of days to future & 148 \\
\hline Current number of days & 63 \\
\hline Enter current value of Ht & 52 \\
\hline
\end{tabular}

www.ijcat.com was more better than the results of Elman neural network, see Table 4.14 for result outputted by the Time-delay neural network when current values of parameters were at 63 days and the expected future values at 148 days. 
International Journal of Computer Applications Technology and Research Volume 8-Issue 06, 229-268, 2019, ISSN:-2319-8656

\begin{tabular}{|l|l|}
\hline Enter current value of G50 & 14 \\
\hline Enter current value of NEL & 8 \\
\hline Enter current value of LW & 32 \\
\hline Enter current value of LL & 63 \\
\hline \multicolumn{2}{|c|}{ The network outputs for 148 days } \\
\hline Predicted value of Ht to future vilue of G50 to future & 182.1825 \\
\hline Predicted value of NEL to future & 45.2846 \\
\hline Predicted value & 25.0498 \\
\hline Predicted value of LW to future & 69.0828 \\
\hline Predicted value of LL to future & 124.3288 \\
\hline Predicted value of BW to future & NIL \\
\hline Predicted value of NHB to future & NIL \\
\hline Predicted value of NFB to future & NIL \\
\hline
\end{tabular}

\subsection{Analysis of Time-delay Neural Network results}

The architecture of the time delay neural network used for the variable $\mathrm{Ht}$, G50, NEL, LW and LL were 1:2:1 and were only trained repeatedly 2 times except for G50 that was trained repeatedly for 3 times. The RMSE and the $\mathrm{R}^{2}$ values selected for all the variables were recorded in Table 4.15.

Table 4.16 shows the Time delay Network Results when DAP was 121 at current and 318 at future values. The result generated and the error values were more reasonable compared to what Elman network outputted. The variation in the predicted values and the experimented, when DAP was 121 at current or present day and 318 at future values of Time delay network and Elman network can be explained from the Table 4.17.

Comparing the RMSE for each variable parameter with the result of the network, it was observed that the variation in the predicted values from the experimental values can be tolerated for each of the parameter for the Time delay network, however the error difference from NFB was much and this could be expected from the architecture selected. The Time delay network predicted more accurately the future values of parameters identified for the plantain plant growth and yield.

Table 4.15: The RMSE and the $\mathrm{R}^{2}$ values for Time delay network architecture selected 
International Journal of Computer Applications Technology and Research

Volume 8-Issue 06, 229-268, 2019, ISSN:-2319-8656

\begin{tabular}{|l|l|l|l|l|l|l|l|l|}
\hline Variable Parameters & Ht & G50 & NEL & LW & LL & NFB \\
\hline Architecture & $1: 2: 1$ & $1: 2: 1$ & $1: 2: 1$ & $1: 2: 1$ & $1: 2: 1$ & $5: 3: 1$ \\
\hline Repeated training times & 2 & 3 & 2 & 2 & 2 & $5: 3: 1$ \\
\hline $\mathrm{R}^{2}$ & 1.000 & 0.9999 & 1.000 & 0.9995 & 0.9993 & 0.2715 \\
\hline RMSE & 0.6005 & 0.1756 & 0.0475 & 0.3524 & 1.1660 & 3.2806 & 0.5391 \\
\hline
\end{tabular}


Table 4.16: Time delay Network Results when DAP was 121 at current and 318 at future values

\begin{tabular}{|l|l|}
\hline \multicolumn{2}{|c|}{ Known values to network } \\
\hline Enter the number of days to future & 318 \\
\hline Current number of days & 121 \\
\hline Enter current value of Ht & 162 \\
\hline Enter current value of G50 & 37.4 \\
\hline Enter current value of NEL & 20 \\
\hline Enter current value of LW & 70 \\
\hline Enter current value of LL & 146 \\
\hline \multicolumn{1}{|c|}{ The network outputs for 318 days } & 405.0015 \\
\hline Predicted value of Ht to future & 74.3601 \\
\hline Predicted value of G50 to future & 38.3278 \\
\hline Predicted value of NEL to future & 76.698 \\
\hline Predicted value of LW to future & 155.4723 \\
\hline Predicted value of LL to future & 21.8758 \\
\hline Predicted value of BW to future & 10.3096 \\
\hline Predicted value of NHB to future & 142.0251 \\
\hline Predicted value of NFB to future & \\
\hline
\end{tabular}

Table 4.17: The variation in the predicted values and the experimented when DAP was 121 at current and 318 at future values of Time delay network and Elman Network

\begin{tabular}{|l|l|l|l|l|l|l|}
\hline \multicolumn{1}{|c|}{$\begin{array}{c}\text { Variable } \\
\text { Parameters }\end{array}$} & \multicolumn{1}{|c|}{$\begin{array}{c}\text { Experimented } \\
\text { values }\end{array}$} & \multicolumn{1}{c|}{$\begin{array}{c}\text { Predicted } \\
\text { values }\end{array}$} & \multicolumn{1}{c|}{ Difference } & \multicolumn{1}{c|}{ Predicted values } & Difference \\
\hline & \multicolumn{2}{|c|}{ Elman Network } & \multicolumn{2}{c|}{ Time delay Network } \\
\hline Ht (cm) & 400 & 476.0604 & 76.0604 & 405.0015 & 7.0015 \\
\hline G50 (cm) & 74 & 31.2002 & 42.7998 & 74.3601 & 0.3601 \\
\hline NEL (unit) & 37 & 5.4523 & 31 & 38.3278 & 1.3278 \\
\hline LW (cm) & 81 & 129.863 & 48.863 & 76.698 & 4.302 \\
\hline LL (cm) & 234 & 296.9355 & 62.9355 & 155.4723 & 78.5277 \\
\hline BW (Kg) & 26 & 49.7751 & 23.7751 & 21.8758 & 4.1242 \\
\hline NHB (unit) & 7 & 7.0331 & 0.0331 & 10.3096 & 3.3096 \\
\hline NFB (unit) & 80 & 123.4779 & 43.4779 & 142.0251 & 62.0251 \\
\hline
\end{tabular}




\section{SUMMARY}

This research developed a scientific method using artificial neural network to predict the development growth and yield of plantain plant. Elman time series neural network, a back-propagation algorithm, with 1 input neuron and 1 output neuron with varying number of neurons in the hidden layer was employed in this research to train the parameters identified for plantain growth development. Each of these parameters, the $\mathrm{Ht}$, G50, NEL, LW and LL, were tested on different architecture by varying the number of neurons in the hidden layer to know the best architecture to use. The results of these networks were used to train the $\mathrm{BW}$, NHB and NFB networks. Some predicted results generated by the neural network can still be accepted as the variation in the predicted value and the experimented value are not too much. RMSE was used to know how much the predicted values deviate from the experimental values in the data set on average. The metric unit is the same unit as its variable and the predicted values by the ANNs against the experimental data were used to calculate the coefficients of determination $\left(\mathrm{R}^{2}\right)$.

Another network, Time delay neural network was employed and the result of this network was more accurate than the result of the Elman neural network even with having the dataset of different varieties of plantain plants together for training.

\section{CONCLUSION}

With this scientific approach for predicting the growth and yield of plantain plant, famers or plantain producers can have better conditions for planning, maximizing the efficiency of the business process without loses because it will help to plan their planting if they know the Pseudostem height of the plant sucker, $\mathrm{Ht}(\mathrm{cm})$; Pseudostem girth at $50 \mathrm{~cm}$ above soil level, G50 (cm); Number of emitted leaves on the sucker, NEL; Leaf width, LW $(\mathrm{cm})$; and the Leaf length, LL $(\mathrm{cm})$ to predict into future, the values of Pseudostem height, Ht $(\mathrm{cm})$; Pseudostem girth at $50 \mathrm{~cm}$ above soil level, G50 (cm); Number of emitted leaves, NEL; Leaf width, LW (cm); Leaf length, LL (cm); Bunch weight, BW (Kg); Number of hands in the bunch, NHB and Number of fingers in the bunch, NFB of plantain plant based on the planting conditions for dataset used for training for this research. They would already know the outcome of the production and can forecast the financial expenses plan before and after which will reduce the waste of resources and increase the agricultural economy of the country if implemented. It was evidence that the neural network was able to predict future values of plantain plant growth and the yield which can help the plantain farmers to know their expected farm output before they even go into the farming. If there are enough dataset to train the network for each variety of plantain plant, there could be more accuracy in the result than having all the varieties together. 


\section{REFERENCES}

[1] Aji A, Munajat Q, Pratama A, Kalamullah H, Aprinaldi., Setiyawan J, Arymurthy A (2013).

Detection of Palm Oil Leaf Disease with Image Processing and Neural Network Classification on Mobile Device. IJCTE-5-(3):528-532. doi:10.7763/IJCTE.2013.V5.743

[2] Bala, B.K., Ashraf, M.A., Uddin, M.A. and Janjai, S. (2005). Experimental and neural network prediction of the performance of a solar tunnel drier for a solar drying jack fruit bulbs and leather.J. Food Proc. Eng. 28, 552-566.

[3] Chandler, S. (1995). The nutritional value of bananas. Bananas and Plantains (S. Gowen,ed.) Chapman \& Hall, UK. pp: 486-480

[4] Diamantopoulou, M.J. (2005). Artificial neural networks as an alternative tool in pine bark volume estimation. Computers and Electronics in Agriculture. 48, 235-244.

[5] Fukushima, K., Miyake, S., and Ito, T. (1983) Neocognitron: A neural network model for a mechanism of visual pattern recognition. IEEE Transactions on Systems, Man, and Cybernetics, 13(5):826834.

[6] Gemtos, T. A., Markinos, A. and Nassiou, T. (2005). Cotton Lint Quality Spatial Variability and Correlation with Soil Properties and Yield. Papers presented at the 5th European Conference on Precision Agiculture, Uppsala, Sweden, pp. 361-368.

[7] Grossberg, S. (1976). Adaptive pattern classification and universal recoding, I: Parallel development and coding of neural feature detectors. Biological Cybernetics, 23:121-134.

[8] Hebb, D. O. (1949). The Organization of Behavior: A Neuropsychological Theory. Wiley,New York.

[9] Hopfield, J. J. (1982). Neural networks and physical systems with emergent collective computational abilities. Proc. of the National Academy of Science, USA, 79:2554-2558.

[10] Jiang, S.D., Jiang, D., Yang, X., Clinton, N. and Wang, N. (2004). An artificial neural network model for estimating crop yields using remotely sensed information. Int. J. Remote Sens. 25, 1723-1732.

[11] Kaul, M., Hill, R. L. and Walthall, C. (2005). Artificial Neural Networks for Corn and Soybean Yield Prediction. Agric. Sys., 85: 1- 18.

[12] Khakural, B. R., Robert, P. C. and Huggins, D. R. (1999). Variability of Corn/Soybean Yield and Soil/Landscape Properties across a Southwestern Minnesota Landscape. Precision Agriculture, 573-579.

[13] Khazaei, N.B., Tavakoli, T., Ghassemian, H., Khoshtaghazaa, M.H., Banakar, A. (2013). Applied machine vision and artificial neural network for modeling and controlling of the grape drying process. Computers and Electronics in Agriculture 98, 205 - 213.

[14] Kominakis, A. P., Abas, Z., Maltaris, I. and Rogdakis, E. (2002). A Preliminary Study of the Application of Artificial Neural Networks to Prediction of Milk Yield in Dairy Sheep. Computers and Electronics in Agriculture, 35: 35-48.

[15] Liu, X., He, D., Song, J., and Yang, H. (2010). Research of virtual plant growth model based on neural network. Computer Science and Education (ICCSE), International Conference. https://doi.org/10.1109/ICCSE.2010.5593760

[16] Malsburg, C.V. (1973) Self-organizing of orientation sensitive cells in striate cortex. Kybernetik, 14:85-100.

[17] Mathworks (2018). Elmannet. Retrieved $30^{\text {th }}$ October, 2018 from https://www.mathworks.com/help/deeplearning/ref/elmannet.html?searchHighlight

$=$ elmannet\&s_tid=doc_srchtitle

[18] McCulloch, W.S. and Pitts, W. (1943). A logical calculus of the ideas immanent in nervous activity. Bulletin of Mathematical Biology, 5(4):115-133.

[19] Minsky, M. and Papert, S. (1969). Perceptrons: An Introduction to computational Geometry. MIT Press, Cambridge, Mass.

[20] Movagharnejad, K., and Nikzad, M. (2007). Modelling of tomato drying using artificial neural network. Computers and Electronics in Agriculture. 59: 78-85.

[21] Ngo-Samnick, E.L. (2011). Improved Plantain Production. Cameroon (ISF Cameroun) and The Technical Centre for Agricultural and Rural Cooperation (CTA) 
[22] Papageorgiou, E. I., Aggelopoulou, K. D., Gemtos, T. A. and Nanos, G. D. (2013). Yield Prediction in Apples Using Fuzzy Cognitive Map Learning Approach. Computers and Electronics in Agriculture, 91:19-29.

[23] Papageorgiou, E. I., Markinos, A. T. and Gemtos, T. A. (2011). Fuzzy Cognitive Map Based Approach for Predicting Yield in Cotton Crop Production as a Basis for Decision Support System in Precision Agriculture Application. Appl. Soft Computing, 11: 3643-3657.

[24] Qiao, D.M., Shi, H.B., Pang, H.B., Qi, X.B. and Plauborg, F. (2010). Estimating plant root water uptake using a neural network approach, Agricultural Water Management 98 (2) 251-260.

[25] Rosenblatt F. (1961). Principles of Neurodynamics: Perceptions and the Theory of Brain Mechanism. Spartan Books, Washington DC.

[26] Rumelhart, D., Hinton, G. and Williams, R. (1986). Learning representations by back propagating errors. Nature, 323:533-536. doi:10.1038/323533a0

[27] Safa, M., Samarasinghe, S. and Nejat, M. (2015). Prediction of Wheat Production Using Artificial Neural Networks and Investigating Indirect Factors Affecting It: Case Study in Canterbury Province, New Zealand. Journal of Agriculture, Science and Technology. Vol. 17: 791-803

[28] Sharma, A. K., Sharma, R. K. and Kasana, H. S. (2007). Prediction of First Lactation 305 day Milk Yield in Karan Fries Dairy Cattle Using ANN Modeling. Appl. Soft Computing, 7:1112-1120.

[29] Soares, J. D. R., Pasqual, M., Lacerda, W.S., Silva, S. O. and Donato, S. L. R. (2013). Utilization of Artificial Neural Networks in the Prediction of the Bunches' Weight in Banana Plants. Scientia Horticulturae, 155: 2429.

[30] Sylvain, D., Frédéric, T., Médard, T., Désirée, F., David, E., Jean-Pierre, E., Bernard, K. and Thierry, L. (2018). The 'Plantain-Optim' dataset: Agronomic traits of 405 plantains every 15 days from planting to harvest. https://doi.org/10.1016/j.dib.2018.01.065

[31] Uno, Y., Prasher, S.O., Lacroix, R., Goel, P.K., Karimi, Y., Viau, A. and Patel, R.M. (2005). Artificial neural networks to predict corn yield from compact airborne spectrographic imager data. Comp. Elect. Agric. 47: 149-161.

[32] Werbos, P.J. (1974) Beyond Regression: New Tools for Prediction and Analysis in the Behavioral Sciences. PhD thesis, Harvard University, 1974.

[33] Yusufu, P.A., Mosiko, T.B. and Ojuko, O.O. (2014). Effect of Firm Ripe Plantain Fruit Flour Addition on the Chemical, Sensory and Microbial Quality of Fura Powder. NIFOJ Vol. 32(1), pp: 38 - 44. https://doi.org/10.1016/S0189-7241(15)30094-1

[34] Zee, F. and Bubenheim, D. (1997). Plant Growth Model Using Artificial Neural Networks. https://doi.org/10.4271/972494

[35] Zhang, W., Bai, X.C. and Liu, G. (2007). Neural network modeling of ecosystems: a case study on cabbage growth system. Ecol. Model 201, 3-4.

[36] Zurada, M. J. (1992). Introduction to Artificial Neural Systems. West Publishing Company, New York. 